\title{
Discovery and Validation of Pyridoxic Acid and Homovanillic Acid as Novel Endogenous Plasma Biomarkers of Organic Anion Transporter (OAT) 1 and OAT3 in Cynomolgus Monkeys ${ }^{\circledR}$
}

Hong Shen, David M. Nelson, Regina V. Oliveira, Yueping Zhang, Colleen A. Mcnaney, Xiaomei Gu, Weiqi Chen, Ching Su, Michael D. Reily, Petia A. Shipkova, Jinping Gan, Yurong Lai, Punit Marathe, and W. Griffith Humphreys

Departments of Metabolism and Pharmacokinetics (H.S., Y.Z., X.G., W.C., J.G., Y.L., P.M., W.G.H.), Discovery Toxicology (D.M.N.), Bioanalytical and Discovery Analytical Sciences (R.V.O., C.A.M., M.D.R., P.A.S.), and Discovery Pharmaceutics (C.S.), Pharmaceutical Candidate Optimization, Bristol-Myers Squibb Research and Development, Princeton, New Jersey

Received July 14, 2017; accepted November 17, 2017

\section{ABSTRACT}

Perturbation of organic anion transporter (OAT) 1- and OAT3mediated transport can alter the exposure, efficacy, and safety of drugs. Although there have been reports of the endogenous biomarkers for OAT1/3, none of these have all of the characteristics required for a clinical useful biomarker. Cynomolgus monkeys were treated with intravenous probenecid (PROB) at a dose of $40 \mathrm{mg} / \mathrm{kg}$ in this study. As expected, PROB increased the area under the plasma concentration-time curve (AUC) of coadministered furosemide, a known substrate of OAT1 and OAT3, by 4.1-fold, consistent with the values reported in humans (3.1- to 3.7-fold). Of the 233 plasma metabolites analyzed using a liquid chromatography-tandem mass spectrometry (LC-MS/MS)-based metabolomics method, 29 metabolites, including pyridoxic acid (PDA) and homovanillic acid (HVA), were significantly increased after either 1 or $\mathbf{3}$ hours in plasma from the monkeys pretreated with PROB compared with the treated animals. The plasma of animals was then subjected to targeted LC-MS/MS analysis, which confirmed that the PDA and HVA AUCs increased by approximately 2- to 3-fold by PROB pretreatments. PROB also increased the plasma concentrations of hexadecanedioic acid (HDA) and tetradecanedioic acid (TDA), although the increases were not statistically significant. Moreover, transporter profiling assessed using stable cell lines constitutively expressing transporters demonstrated that PDA and HVA are substrates for human OAT1, OAT3, OAT2 (HVA), and OAT4 (PDA), but not OCT2, MATE1, MATE2K, OATP1B1, OATP1B3, and sodium taurocholate cotransporting polypeptide. Collectively, these findings suggest that PDA and HVA might serve as blood-based endogenous probes of cynomolgus monkey OAT1 and OAT3, and investigation of PDA and HVA as circulating endogenous biomarkers of human OAT1 and OAT3 function is warranted.
Introduction

The organic anion transporters (OATs) OAT1 (SLC22A6) and OAT3 (SLC22A8) function as influx transporters that are mainly expressed on the basolateral membrane of renal proximal tubular cells (RPTCs) and mediate cellular uptake of substrates from blood into the cells (Motohashi et al., 2002). OAT1 and OAT3 can serve as the loci of drug-drug interactions (DDIs), and such DDIs can lead to undesired changes in systemic and local exposures of victim drugs and toxins (Morrissey et al., 2013; Nigam et al., 2015). For example, furosemide (FSM) is taken up from the blood in RPTCs via OAT1 and OAT3 (Hasannejad et al., 2004), and probenecid (PROB), an inhibitor of OAT1

This study is supported by the Bristol-Myers Squibb Company. https://doi.org/10.1124/dmd.117.077586.

S This article has supplemental material available at dmd.aspetjournals.org. and OAT3, causes significant alterations in the pharmacokinetic parameters of FSM in humans through the increased area under the plasma drug concentration-time curve (AUC), decreased total plasma clearance $\left(\mathrm{CL}_{\mathrm{TOT}}\right)$ and renal clearance $\left(\mathrm{CL}_{\mathrm{R}}\right)$, and decreased fraction excreted in the urine $\left(f_{\mathrm{R}}\right)$ (Chennavasin et al., 1979; Smith et al., 1980; Vree et al., 1995). Moreover, a few uremic toxins recently have been demonstrated to be inhibitors of OAT1 and OAT3, and have the potential to inhibit renal secretion clearance of drug substrates of OAT1 and OAT3 in patients with chronic kidney disease (Hsueh et al., 2016). On the other hand, concomitant use of PROB is recommended by the U.S. Food and Drug Administration for reducing the pronounced nephrotoxicity of cidofovir by inhibiting OAT1 and OAT3 to reduce the exposure of cidofovir in RPTCs to an extent that results in an acceptable benefitrisk balance (http://www.accessdata.fda.gov/drugsatfda_docs/label/ 1999/020638s003lbl.pdf).

ABBREVIATIONS: ACN, acetonitrile; $\mathrm{AUC}$, area under the plasma concentration-time curve; $\mathrm{BSA}$, bovine serum albumin; $\mathrm{CL}_{\mathrm{NR}}$, nonrenal plasma clearance; $\mathrm{CL}_{R}$, renal clearance; $\mathrm{CL}_{\mathrm{TO}}$, total plasma clearance; DDI, drug-drug interaction; Enal-d5, enalapril maleate-d5; FSM, furosemide; HBSS, Hanks' balanced salt solution; HDA, hexadecanedioic acid; HEK, human embryonic kidney; HILIC, hydrophilic interaction liquid chromatography; HVA, homovanillic acid; LC, liquid chromatography; LC-MS/MS, liquid chromatography-tandem mass spectrometry; MS, mass spectrometry; NTCP, sodium taurocholate cotransporting polypeptide; OAT, organic anion transporter; PBS, phosphate-buffered saline; PDA, pyridoxic acid; $\mathrm{PROB}$, probenecid; QC, quality control; RP, reverse phase; RPTC, renal proximal tubular cell; $\mathrm{TDA}$, tetradecanedioic acid; $\mathrm{Vd}_{\mathrm{ss}}$, volume of distribution at steady-state. 
Animal models have often been used to assess preclinical drug-OAT interactions. For example, the clinical famotidine-PROB interaction was reproduced in cynomolgus monkeys recently since PROB caused an approximate $90 \%$ reduction in the tubular secretion clearance of famotidine in monkeys ( $4.58 \pm 1.25$ vs. $0.38 \pm 0.36 \mathrm{ml} / \mathrm{min}$ per kilogram) (Tahara et al., 2006). Similarly, PROB significantly decreased the renal tubular secretion clearance of famotidine from $2.80 \pm 0.36$ to $0.37 \pm 0.07 \mathrm{ml} / \mathrm{min}$ per kilogram in humans. Furthermore, the protective effect of PROB treatment on the nephrotoxicity of cidofovir in humans was able to be recapitulated in monkeys (Lacy et al., 1998). These results suggest that monkey is the more suitable animal model to predict the clinical DDIs involving OAT1 and OAT3.

Recently, endogenous biomarkers have been envisioned as simple, fast, and cost-effective tools to monitor transporter activity in preclinical and clinical settings to facilitate development of a drug candidate (Ito et al., 2012; Müller et al., 2015; Lai et al., 2016; Yee et al., 2016; Bergagnini-Kolev et al., 2017). Therefore, the identification of a sensitive endogenous biomarker for OAT1 and OAT3 would be of great value. In this regard, Imamura et al. (2014) reported that $6 \beta$ hydroxycortisol could be an endogenous probe for OAT3 inhibition evaluation since PROB significantly changed the AUC and $\mathrm{CL}_{\mathrm{R}}$ of $6 \beta$ hydroxycortisol in healthy subjects. However, $6 \beta$-hydroxycortisol is formed from cortisol by hepatic CYP3A4, and many drugs known to affect this drug-metabolizing enzyme can also change exposure of $6 \beta$ hydroxycortisol (Peng et al., 2011). Very recently, Tsuruya et al. (2016) reported that taurine and glycochenodeoxycholate sulfate were endogenous biomarkers of OAT1 and OAT3, respectively. However, the plasma levels of taurine and glycochenodeoxycholate sulfate were not changed significantly by PROB treatment compared with control even though the renal secretion and $C_{R}$ were significantly decreased (Tsuruya et al., 2016). Reduced activities of blood-facing OAT1 and OAT3 by PROB are supposed to increase plasma concentration of a sensitive and selective endogenous probe, mimicking systemic alterations of probe drugs such as FSM. To our knowledge, all published endogenous biomarkers of OAT1 and OAT3 could not recapitulate plasma drug concentration-time profiles in the presence of OAT inhibitors. Several features including specificity, sensitivity, predictability, reproducibility, acute response, and accessibility have been considered in the identification and validation of ideal endogenous biomarkers for drug transporters (Chu et al., 2017; Mariappan et al., 2017; Rodrigues et al., 2017). Given the aforementioned reasons, there is a need for novel plasma biomarkers of renal OATs.

In the present studies we provide direct experimental evidence that several organic anionic compounds, including pyridoxic acid (PDA), homovanilic acid (HVA), hexadecanedioic acid (HDA), and tetradecanedioic acid (TDA) are potential endogenous biomarkers of OAT1 and OAT3 in cynomolgus monkeys. Specifically, an untargeted metabolomics analysis was applied to plasma samples to screen endogenous compounds that were associated with OAT1 and OAT3 inhibition in monkeys. Follow-up quantitative liquid chromatography-tandem mass spectrometry (LC-MS/MS) analysis further characterized the timeplasma concentration profiles of selected endogenous compounds (i.e., PDA, HVA, had, and TDA) after PROB administration in cynomolgus monkeys. Moreover, transporter profiling assessed using human embryonic kidney (HEK) 293 cells stably transfected with major human renal and hepatic drug transporters demonstrated that PDA and HVA are OAT1 and OAT3 substrates and thus potential novel plasma endogenous biomarkers of OAT1 and OAT3 inhibition.

\section{Materials and Methods}

Materials. $\left[{ }^{3} \mathrm{H}\right]$ Penciclovir $(1.3 \mathrm{Ci} / \mathrm{mmol})$ and $\left[{ }^{14} \mathrm{C}\right] \mathrm{metformin}(98 \mathrm{mCi} / \mathrm{mmol})$ were purchased from Moravek Biochemicals, Inc. (Brea, CA), and $\left[{ }^{3} \mathrm{H}\right]$ para- aminohippuric acid $(4.5 \mathrm{Ci} / \mathrm{mmol}),\left[{ }^{3} \mathrm{H}\right]$ estrone-3-sulfate $(44.0 \mathrm{Ci} / \mathrm{mmol}),\left[{ }^{3} \mathrm{H}\right]-$ estradiol-17 $\beta$-D-glucuronide $(34.3 \mathrm{Ci} / \mathrm{mmol})$, and $\left[{ }^{3} \mathrm{H}\right]$ cholecystokinin octapeptide $(97.5 \mathrm{mCi} / \mathrm{mmol})$ were purchased from PerkinElmer Life and Analytical Sciences (Waltham, MA). Nonradiolabeled FSM, PROB, and taurocholic acid, and the corresponding stable isotope-labeled internal standards were purchased from Toronto Research Chemicals Inc. (North York, Ontario, Canada). 4-PDA ( $\geq 98 \%$ ) was purchased from Sigma-Aldrich (St. Louis, MO), and HVA ( $\geq 98 \%)$ was purchased from Acros Organics (Geel, Belgium). Hexadecanedioic acid-d28, tetradecanedioic acid-d24, and enalapril maleate-d5 (Enal-d5) were obtained from CDN Isotopes (Pointe-Claire, Quebec, Canada). Other nonradiolabeled compounds were purchased from either Sigma-Aldrich or Toronto Research Chemicals Inc. and were of analytical grade. Cell culture media and reagents were purchased from Invitrogen (Carlsbad, CA) or Mediatech, Inc., A Corning Subsidiary (Manassas, VA).

Cynomolgus Monkeys Pharmacokinetic FSM-PROB Interaction Study Protocol. To identify and verify the endogenous biomarkers of OAT1 and OAT3, we conducted a series of experiments: cynomolgus monkey FSM-PROB interaction, metabolomics, targeted LC tandem MS (LC-MS/MS), and transporter profiling experiments. The experimental workflow is shown in Fig. 1.

A single-dose, three-period crossover intravenous pharmacokinetic DDI study was carried out at the Bristol-Myers Squibb Company, and three male cynomolgus monkeys with body weights ranging from 5.3 to $6.0 \mathrm{~kg}$ during the study periods were included in this study. The experiment was performed in accordance with the National Institutes of Health guidelines (https://grants.nih. gov/grants/olaw/guide-for-the-care-and-use-of-laboratory-animals.pdf) and approved by the Bristol-Myers Squibb Animal Care and Use Committee. The animals were housed in a temperature- and humidity-controlled room with a 12-hour light/dark cycle.

In the first period, $40 \mathrm{mg} / \mathrm{kg}$ PROB dissolved in a sodium hydroxide solution $(0.1 \mathrm{~N})$ and titrated to neutral $\mathrm{pH}$ with hydrochloric acid was intravenously infused via a femoral vein catheter over 5 minutes $(5 \mathrm{ml} / \mathrm{kg})$. Venous blood samples ( $2 \mathrm{ml})$ were collected before and 0.08, 0.17, 0.25, 0.5, 0.75, 1, 2, 3, 5, 7, and 24 hours after administration in $\mathrm{K}_{2}$-EDTA-containing tubes. In the second period, after a washout period of 7 days, $2 \mathrm{mg} / \mathrm{kg}$ FSM dissolved in saline was intravenously infused via a femoral vein over 5 minutes $(5 \mathrm{ml} / \mathrm{kg})$, and blood samples were collected before and 0.08, 0.17, 0.25, 0.5, 0.75, 1, 2, 3, 5, 7, and 24 hours after administration. In the third period, 8 days after administrations of the previous dose of FSM, a FSM saline solution $(2 \mathrm{mg} / \mathrm{kg}$ ) was given by femoral vein infusion over 5 minutes to each monkey 30 minutes after PROB administration ( $40 \mathrm{mg} / \mathrm{kg}$, i.v. infusion for 5 minutes). Blood was sampled before and $0.08,0.17,0.25,0.5,0.75,1,2,3,5,7$, and 24 hours after start of the FSM infusion. Blood samples were spun for 5 minutes at 13,000 rpm within 1 hour to obtain plasma. Urine samples were collected using metabolic cages for the following intervals in all three periods: $0-3,3-7$, and 7-24 hours after administration, and the volume of urine was recorded. The plasma and urine samples were stored at $-80^{\circ} \mathrm{C}$ until LC-MS/MS and metabolomics analyses were conducted.

LC-MS/MS Analysis of Furosemide and Probenecid. The LC-MS/MS analysis was performed on a SCIEX Triple Quad API-4000 system (AB SCIEX, Framingham, MA) coupled with a Shimadzu Nexera LD-30AD ultraperformance LC system (Shimadzu, Columbia, MD). The chromatographic separation was performed on an Agilent Zorbax RRHD SB-C8 column $(2.0 \times$ $100 \mathrm{~mm}, 1.7 \mu \mathrm{m}$ ) from Agilent Technologies (Santa Clara, CA) using mobile phases of $0.1 \%$ formic acid in water and $0.1 \%$ formic acid in acetonitrile (ACN). The flow rate was $0.7 \mathrm{ml} / \mathrm{min}$ and total run time was 3.7 minutes. The $\mathrm{LC}$ column was maintained at $60^{\circ} \mathrm{C}$. The analytes were monitored using selected reaction monitoring in negative ionization mode with the optimized nebulizing and desolvation gases. The source temperature was set at $400^{\circ} \mathrm{C}$, and the declustering potential and collision energy were optimized. FSM, furosemide-d5, and PROB were detected at the selected reaction monitoring transitions of $\mathrm{m} / \mathrm{z}, 329.1 \rightarrow$ $285.0,334.1 \rightarrow 291.0$, and $283.9 \rightarrow 239.9$, respectively.

Before the analysis, urine samples were diluted 100 -fold into blank plasma and treated as previously described for plasma. The plasma and diluted urine samples were then extracted using protein precipitation. Specifically, the $100 \mu \mathrm{l}$ plasma and diluted urine samples were mixed with $100 \mu$ of ACN containing $400 \mathrm{nM}$ of furosemide-d5 (internal standard), followed by vortex mixing on a mixer for 5 minutes at room temperature. The mixed solutions were then filtered using a MultiScreen hydrophilic flitration plate (Billerica, Millipore, MA) by 


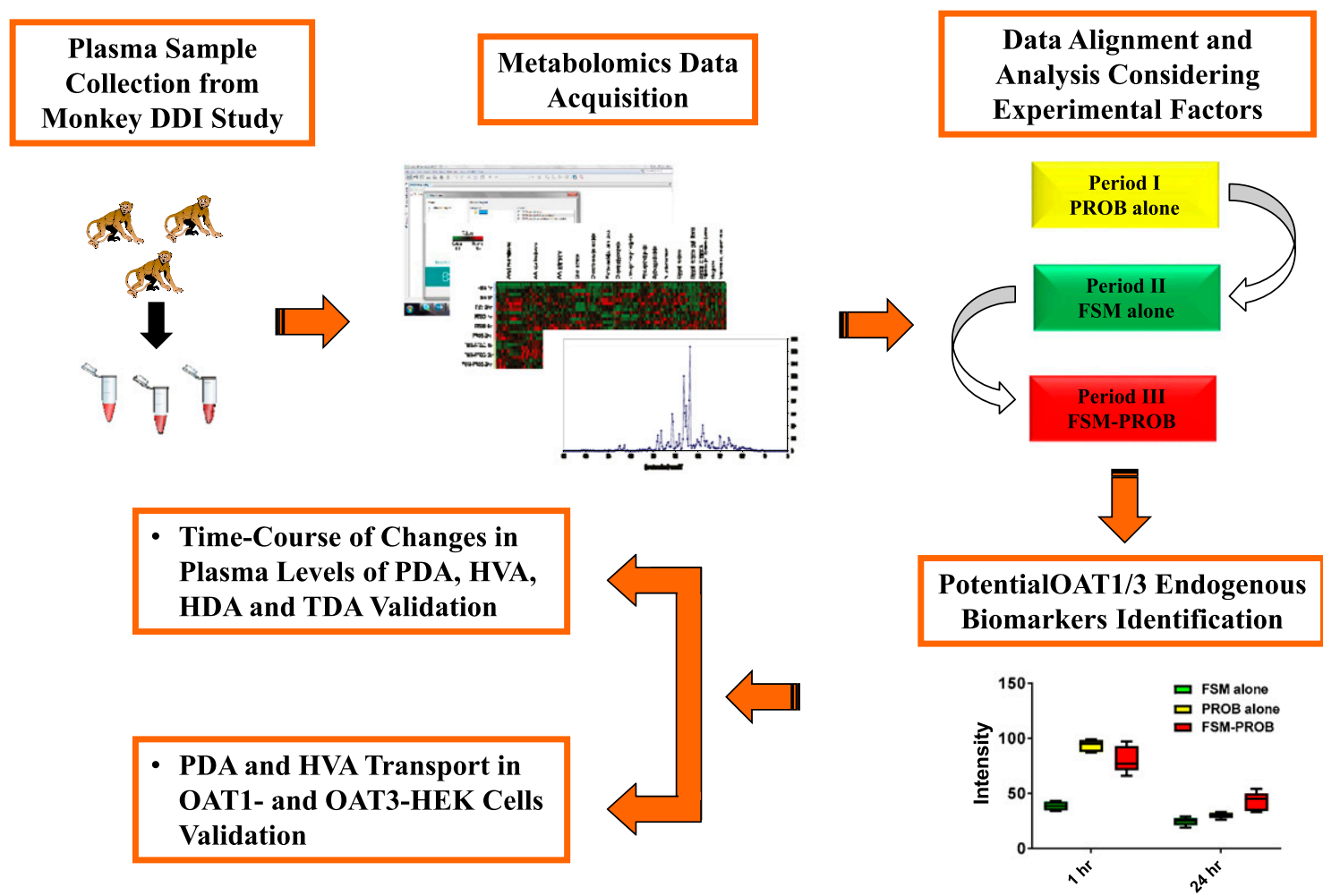

Fig. 1. Schematic overview of experimental workflow for identification and validation of endogenous biomarkers of OAT1 and OAT3. Plasma samples were collected from monkey FSM-PROB interaction study, and metabolites were quantified by LC-MS/MS. Raw data were extracted, and analyzed by various tools to identify associations between drug treatments and concentrations, determine significant correlations, and integrate results with transporter knowledge. Three selected potential endogenous biomarkers were further validated by LC-MS/MS with deuterated internal standards to confirm metabolomics observations. Transporter uptake studies were performed to determine whether the selected probes were selective substrates for human OAT1 and OAT3.

centrifugation at $4000 \mathrm{~g}$ for 10 minutes, and the filtered solution was then injected $(5 \mu \mathrm{l})$ onto LC-MS/MS. The assay was qualified over the analytical range of $1-5000 \mathrm{nM}$ using a linear $1 / x^{2}$ weighed regression.

Plasma Metabolomic Profiling. Frozen plasma samples were thawed and $50 \mu \mathrm{l}$ aliquots were subjected to protein precipitation by the addition of $600 \mu \mathrm{l}$ of methanol containing $0.1 \%$ formic acid and stable-labeled internal standards. The samples were mixed with a vortex mixer and subjected to centrifugation for 10 minutes at $2700 \mathrm{~g}(4000 \mathrm{rpm})$. An aliquot $(50 \mu \mathrm{l})$ of the resulting supernatants was transferred to a 96-well plate for further hydrophilic interaction LC (HILIC) LC-MS analysis and an additional $100 \mu \mathrm{l}$ was transferred to a separate 96-well plate for reverse phase (RP) LC-MS analysis. Both plates were dried to completeness under a nitrogen stream at room temperature. For RP LC-MS analysis, the dried samples were reconstituted by the addition of $20 \mu \mathrm{l}$ of methanol, followed by vigorous shaking and the addition of $180 \mu \mathrm{l}$ of water. The samples were mixed with a vortex mixer and subjected to centrifugation for 10 minutes at $4000 \mathrm{rpm}$. The resulting supernatants were transferred to a 96-well plate, from which $10 \mu \mathrm{l}$ was directly injected for analysis. For HILIC LC-MS analysis, the dried samples were reconstituted by the addition of $20 \mu \mathrm{l}$ of water, followed by vigorous shaking and the addition of $180 \mu \mathrm{l}$ of 50:50 MeOH:ACN. The samples were mixed with a vortex mixer and subjected to centrifugation for 10 minutes at $4000 \mathrm{rpm}$. The resulting supernatants were transferred to a 96-well plate, from which $10 \mu \mathrm{l}$ was directly injected for analysis.

HILIC and RP LC-MS analyses were performed on a Nexera $\times 2$ LC-30AD (Shimadzu, Somerset, NJ) ultra-high-performance LC system connected to an Exactive Plus (Thermo Fisher Scientific, Waltham, MA) mass spectrometer. The ultra-high-performance LC column for HILIC analyses was an Acquity BEH$\mathrm{NH} 2,2.1 \times 150 \mathrm{~mm}, 1.7 \mu$ (Waters Corporation, Milford, MA) with mobile

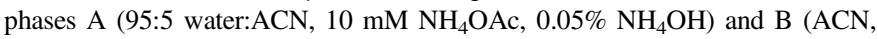
$\left.0.05 \% \mathrm{NH}_{4} \mathrm{OH}\right)$ at a flow rate of $300 \mu \mathrm{l} / \mathrm{min}$ with starting conditions of $95 \% \mathrm{~B}-$ $37 \% \mathrm{~B}$ at 3.5 minutes, hold for 4 minutes, and down to starting conditions at 7 minutes, for a total run time of 11 minutes. The ultra-high-performance LC column for RP analyses was an Acquity BEH C18, $2.1 \times 150 \mathrm{~mm}, 1.7 \mu$ (Waters
Corporation) with mobile phases A (water, $0.1 \%$ formic acid) and B (98:2ACN: water, $0.1 \%$ formic acid) at a flow rate of $600 \mu \mathrm{l} / \mathrm{min}$ with starting conditions of $100 \% \mathrm{~A}$, to $80 \% \mathrm{~A}$ at 3 minutes, $40 \% \mathrm{~A}$ at 4 minutes, and $100 \%$ B by 7 minutes, and after a 2-minute hold, down to starting conditions at 9 minutes, for a total run time of 11 minutes. Both HILIC and RP LC-MS data were collected in positive and negative polarities (separate injections) at 35,000 resolution and expected mass accuracy of $5 \mathrm{ppm}$.

LC-MS data analysis was performed using software developed in-house, Expedient Data Mining, as described previously (Hnatyshyn et al., 2013). Direct data input from raw files was performed using the Thermo Fisher Scientific MSFileReader software. The resulting list of components was matched with accurate mass and retention time values using an in-house database of endogenous metabolites stored within Expedient Data Mining. The annotated table of component integrals was exported to Microsoft Excel for further statistical analysis using in-house Visual Basic scripts for Microsoft Excel. For these analyses, mean intensities for each treatment group were compared with the relevant concurrent control. For each component, fold change was calculated by dividing the treatment group value by the control group value and the $P$ values were calculated as a pairwise comparison for two-tailed distribution, using the Student's $t$ test (Excel statistics package; Microsoft; Redmond, Washington).

Characterization of PDA and HVA Uptake in Stable Cell Lines Constitutively Expressing Major Renal Drug Transporters. Uptake studies were performed as described previously (Shen et al., 2016b). Uptake of PDA and HVA was first measured at a single concentration for 5-minute incubation with OAT1-, OAT2-, OAT3-, OAT4-, OCT2-, MATE1-, MATE2K-, OATP1B1-, OATP1B3-, and sodium taurocholate cotransporting polypeptide (NTCP)-HEK cells, and then time- and concentration-dependent uptake was measured. The initial concentration used for PDA was $1 \mu \mathrm{M}$ because the physiologic baseline level ranged from 0.67 to $2.5 \mu \mathrm{M}$ in cynomolgus monkeys (Fig. 3A). The concentration studied for HVA was $5 \mu \mathrm{M}$ because of limited bioanalytical sensitivity of this compound in Mock-HEK cells. In addition, the transport Michaelis-Menten constant value $\left(K_{\mathrm{m}}\right)$ of $274 \pm 100 \mu \mathrm{M}$ (that corresponds to the 
substrate concentration at which the uptake rate is half that of the $V_{\max }$ value) has been reported in rat Oat2-mediated transport for HVA (Mori et al., 2003), which is significantly greater than the selected testing concentrations $(274 \pm 100$ vs. $5 \mu \mathrm{M})$. No corresponding data were available for PDA. Kinetic transport experiments were conducted under linear-uptake conditions or for shortest incubation duration with acceptable analytical sensitivity (Supplemental Fig. 1).

Cells were grown to confluence in 24-well poly-D-lysine-coated plates 2-3 days after seeding at a cell density of 500,000 cells per well (BD Biosciences, San Jose, CA). All experiments were conducted at $37^{\circ} \mathrm{C}$ using a working solution containing Hanks' balanced salt solution (HBSS) supplemented with $10 \mathrm{mM} \mathrm{HEPES} \mathrm{(pH} 7.4$ for OAT1-, OAT2-, OAT3-, OAT4-, OCT2-, OATP1B1, OATP1B3-, and NTCP-HEK cells, and $\mathrm{pH} 8.4$ for MATE1- and MATE2K-HEK cells, respectively), with a probe substrate, PDA, or HVA. The probe substrates used were $\left[{ }^{3} \mathrm{H}\right]$ para-aminohippuric acid (OAT1), $\left[{ }^{3} \mathrm{H}\right]$ penciclovir (OAT2), $\left[{ }^{3} \mathrm{H}\right]$ estrone-3-sulfate (OAT3 and OAT4), $\left[{ }^{14} \mathrm{C}\right]$ metformin (OCT2, MATE1, and MATE2K), $\left[{ }^{3} \mathrm{H}\right]$ estradiol-17 $\beta$-D-glucuronide (OATP1B1), $\left[{ }^{3} \mathrm{H}\right]$ cholecystokinin octapeptide [OATP1B3, and taurocholic acid (NTCP)]. Compounds were dissolved in dimethyl sulfoxide and diluted in HBSS (maximum $0.2 \%$ dimethyl sulfoxide). Plating medium was removed, and cell monolayers were rinsed twice with prewarmed HBSS. Incubations were started by the addition of $200 \mu \mathrm{l}$ of substrate prewarmed at $37^{\circ} \mathrm{C}$. After incubation for desired time at $37^{\circ} \mathrm{C}$, the cell monolayers were rinsed three times with $500 \mu \mathrm{l}$ of ice-cold HBSS. The cells were then lysed with $300 \mu$ l buffer $(0.1 \%$ Triton X-100 or methanol), and compound concentrations in the cell lysates were measured by either liquid scintillation counting (Tri-Carb 2910 TR Liquid Scintillation Analyzer; PerkinElmer Life and Analytical Sciences) or LC-MS/MS as described subsequently.

LC-MS/MS Analysis of PDA and HVA. Stock PDA solution $(390 \mu \mathrm{g} / \mathrm{ml}$, $2.13 \mathrm{mM}$ ) was prepared by dissolving PDA in $0.5 \% \mathrm{NH}_{4} \mathrm{OH}$ solution. Stock HVA $(1.8 \mathrm{mg} / \mathrm{ml}, 9.5 \mathrm{mM})$ solution was prepared in water. Stock solutions were stored in darkness at $-30^{\circ} \mathrm{C}$ and brought to room temperature before use. The highest calibration standard $(5000 \mathrm{ng} / \mathrm{ml})$ was prepared by diluting appropriate aliquots of PDA and HVA stock solutions to a final volume of $2 \mathrm{ml}$ with $1 \%$ bovine serum albumin (BSA) in phosphate-buffered saline (PBS) (pH 7.4). Additional standard solutions were obtained by serial dilution from the $5000 \mathrm{ng} / \mathrm{ml}$ standard with $1 \%$ BSA in PBS (pH 7.4) to final concentrations of 2500, 1000, 500, 250, 100, 50, 25, 10, and $5 \mathrm{ng} / \mathrm{ml}$. Quality control (QC) samples were also prepared with $1 \%$ BSA in PBS (pH 7.4) at three concentration levels: $7.5,75$, and $750 \mathrm{ng} / \mathrm{ml}$. The internal standard stock solution of Enal-d5 was prepared at $1 \mathrm{mg} / \mathrm{ml}$ in methanol. The internal standard working solution containing $1000 \mathrm{ng} / \mathrm{ml}$ of Enal-d5 was prepared by dilution of internal standard stock solution with methanol and stored at $4{ }^{\circ} \mathrm{C}$.

Calibration curves for PDA and HVA were fitted by the $1 / x$ weighted leastsquares quadratic. The calibration curves for PDA and HVA ranged from 5 to $5000 \mathrm{ng} / \mathrm{ml}$. All coefficients of determination $\left(R^{2}\right)$ of the calibration lines were $\geq 0.98$. The mean accuracy (percentage of true value) of individual calibrators was $\geq 15 \%$. The lower limit of quantitation, defined as the lowest concentration that could be determined with precision and accuracy of $\pm 20 \%$, was $5 \mathrm{ng} / \mathrm{ml}$ for PDA and HVA.

Sample stability was also determined for QC samples $(n=5)$ that were extracted and stored in the instrument autosampler under refrigerated conditions $\left(5^{\circ} \mathrm{C}\right)$. Samples were stable for 24 hours. Average recovery for QC samples $(n=5)$ prepared with $1 \%$ BSA in PBS (pH 7.4) was $101 \%$ and $102 \%$ for PDA and HVA, respectively.

Urine and plasma samples were diluted by factors of 1:1, 1:2, 1:10 or 1:100 with $1 \%$ BSA in PBS (pH 7.4) to ensure the levels were within the range of the calibration curve. Aliquots $(50 \mu \mathrm{l})$ of diluted animal samples (urine and plasma), calibrator standards, or QCs were transferred to a $2 \mathrm{ml}$ 96-well plate and mixed for 1 minute at $2000 \mathrm{rpm}$. The samples were extracted by adding $200 \mu \mathrm{l}$ of Enal-d5 in methanol $(1000 \mathrm{ng} / \mathrm{ml})$ and vortex mixed for 5 minutes at $2000 \mathrm{rpm}$, and then centrifuged at $4^{\circ} \mathrm{C}$ for 10 minutes at $4000 \mathrm{~g}$. The supernatant $(170 \mu \mathrm{l})$ was transferred to a $500 \mu \mathrm{l}$ 96-well plate and dried under heated nitrogen $\left(45^{\circ} \mathrm{C}\right)$. Samples were then reconstituted with $50 \mu \mathrm{l}$ of water:methanol (98:2) containing $0.1 \%$ formic acid, vortex mixed for 1 minute at $2000 \mathrm{rpm}$, followed by 10 minutes of centrifugation at $4000 \mathrm{~g}$ at $4^{\circ} \mathrm{C}$ before MS analysis. To avoid PDA and HVA degradation, all samples were protected from direct light exposure during sample preparation and analysis.

LC-MS/MS analyses were carried out on a Waters Acquity UPLC system, consisting of a Acquity binary solvent manager and Acquity sample manager with sample organizer (Waters) coupled to a SCIEX 6500 tandem quadrupole mass spectrometer (Applied Biosystems/MDS SCIEX, Toronto, Canada) equipped with an electrospray ionization source. The analytes $(10 \mu \mathrm{l})$ were separated on an Acquity UPLC BEH130 C18 $(2.1 \times 100 \mathrm{~mm} ; 1.7 \mu \mathrm{m}$ particle size $)$ column and eluted by a gradient program as follows: held $2 \% \mathrm{~B}$ for 0.5 minutes, $2 \% \mathrm{~B}-20 \% \mathrm{~B}$ for 2.5 minutes, $20 \%$ B-98\% B for 1 minute, $98 \%$ B-2\% B for 0.01 minute, and retained 1 minute for equilibration. The column was heated at $45^{\circ} \mathrm{C}$, and the flow rate was $500 \mu \mathrm{l} / \mathrm{min}$. The mobile phase consisted of an aqueous phase (A: $0.1 \%$ formic acid in water) and an organic phase (B: $0.1 \%$ formic acid in $\mathrm{ACN}$ ).

The electrospray ionization source was operated in negative ion mode and its main working parameters were set as follows: ion spray voltage, $-4.5 \mathrm{kV}$; ion source temperature, $550^{\circ} \mathrm{C}$; declustering potential, $-40 \mathrm{~V}$ for PDA and HVA and $-150 \mathrm{~V}$ for Enal-d5; collision energy, $-35 \mathrm{~V}$; entrance potential, $-10 \mathrm{~V}$; and collision cell exit potential, $-10 \mathrm{~V}$. Multiple reactions monitoring measurements of PDA and HVA analytes were performed using individually optimized cone voltage and collision energy. The multiple reactions monitoring precursor/product ion transitions were as follows: $m / z 182>138.0$ for PDA, $181.0>122.0$ for HVA, and 380.3 $>$ 114.2 for the internal standard, Enal-d5. The dwell time established for each transition was 50 milliseconds. All peak integration and data processing methods were performed using SCIEX Analyst 1.6.2 (Applied Biosystems/MDS SCIEX).

Pharmacokinetic, Transport, and Statistical Analyses. The AUC from zero to 24 hours $\left(\mathrm{AUC}_{0-24 \mathrm{~h}}\right)$ was calculated using the mixed trapezoidal rule. The AUC from zero to infinity includes $\mathrm{AUC}_{0-24 \mathrm{~h}}$ and one extrapolated to infinity from the last measured concentration. The volume of distribution at steady-state $\left(\mathrm{Vd}_{\mathrm{SS}}\right)$ was determined by the noncompartmental method:

$$
\mathrm{Vd}_{\mathrm{ss}}=\frac{\text { Dose } \cdot(\text { AUMC })}{(\mathrm{AUC})^{2}}
$$

where AUMC is the area under the curve of the first moment of the concentrationtime curve. The $\mathrm{CL}_{\text {TOт }}$ was calculated from the following equation:

$$
\mathrm{CL}_{\mathrm{TOT}}=\frac{\text { Dose }}{\mathrm{AUC}}
$$

The pharmacokinetics parameters including $\mathrm{AUC}_{0-24 \mathrm{~h}}, \mathrm{AUC}, \mathrm{Vd}_{\mathrm{SS}}$, and $\mathrm{CL}_{\text {TOT }}$ for FSM, PROB, PDA, and HVA, following single intravenous administration of PROB and FSM with or without coadministration of PROB were analyzed with a mixed trapezoidal model using the Kinetica program (Thermo Electron, Philadelphia, PA). The $\mathrm{CL}_{\mathrm{R}}$ was estimated from the following equation:

$$
\mathrm{CL}_{\mathrm{R}}=\frac{\mathrm{Xe}_{0-24 \mathrm{~h}}}{\mathrm{AUC}_{0-24 \mathrm{~h}}}
$$

where $\mathrm{Xe}_{0-24} \mathrm{~h}$ is the cumulative amount of unchanged FSM excreted in urine over 24 hours. The renal extraction ratio $\left(\mathrm{ER}_{\mathrm{R}}\right)$ of FSM was calculated by the following equation:

$$
\mathrm{ER}_{\mathrm{R}}=\frac{\mathrm{CL}_{\mathrm{R}}}{f_{\mathrm{u}} \cdot \mathrm{GFR}}
$$

where $f_{\mathrm{u}}$ is the fraction of unbound FSM in human plasma reported (i.e., 0.041) (Rane et al., 1978), and GFR is the glomerular filtration rate in cynomolgus monkeys (i.e., $10.4 \mathrm{ml} / \mathrm{min}$ ) (Davies and Morris, 1993). The fraction of FSM excreted unchanged in the urine $\left(f_{\mathrm{R}}\right)$ was calculated by dividing $\mathrm{Xe}_{0-24 \mathrm{~h}}$ by the dose. The nonrenal plasma clearance $\left(\mathrm{CL}_{\mathrm{NR}}\right)$ was estimated as the difference between the total plasma and renal clearances. The fraction excreted by nonrenal routes $\left(f_{\mathrm{NR}}\right)$ was calculated by dividing the nonrenal clearance by the total plasma clearance. Paired Student's $t$ test was performed to compare pharmacokinetic parameters between groups using GraphPad Prism version 7 (GraphPad Software Inc., San Diego, CA). A $P$ value of less than 0.05 was considered to be statistically significant $(* P<0.05$, $* * P<0.01$, and $* * * P<0.001)$. To compare pharmacokinetic parameters between groups, the data are also reported as the geometric mean ratio with a two-sided $90 \%$ confidence interval.

Transport data represent the results from a single study run in triplicate and a minimum of two experiments on different days. The results are reported as mean \pm S.D. $(n=3)$. To estimate the transport kinetics parameters of PDA and HVA into transporter-expressing HEK 293 cells, the transporter-mediated uptake was calculated by subtracting the uptake in MOCK-HEK cells from that in transporter-expressing HEK 293 cells. The following equation was used to estimate the parameters:

$$
V=\frac{V_{\max } \times[S]}{K_{\mathrm{m}}+[S]}
$$


A

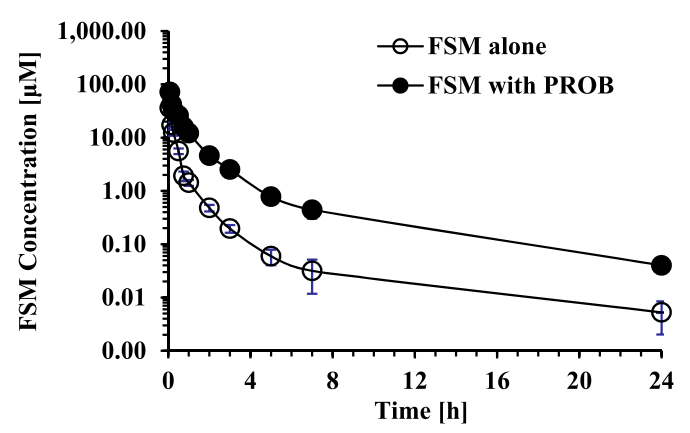

B

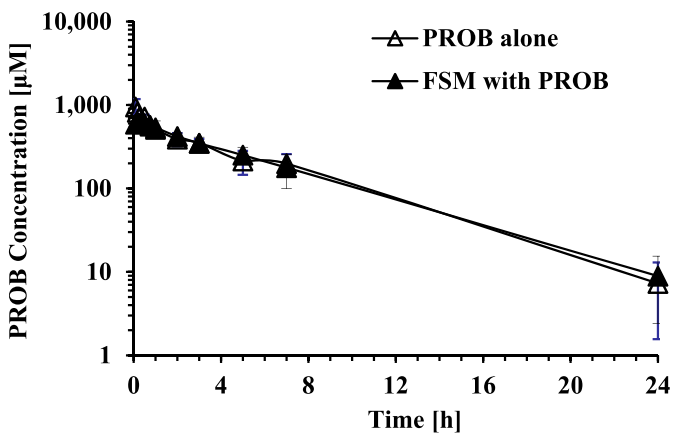

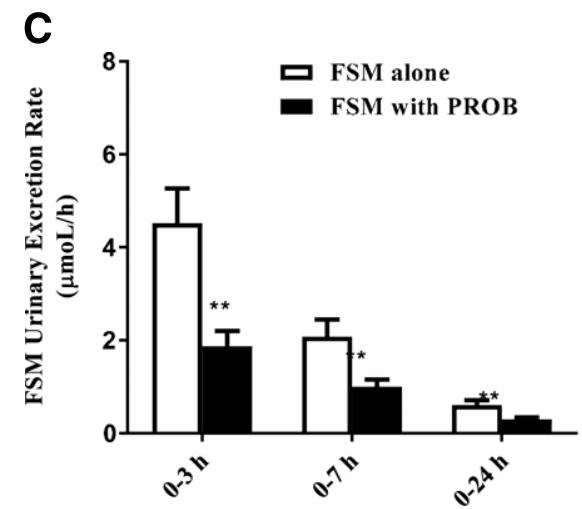

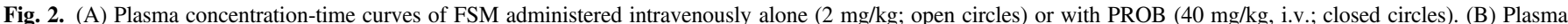

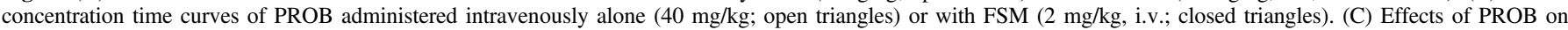

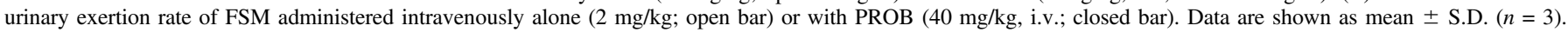
$* * P<0.01$, significantly different from urinary excretion rate in the absence of PROB.

where $V$ is the rate of uptake measured at the given concentration; $V_{\max }$ is the maximal rate of uptake; $K_{\mathrm{m}}$ represents the Michaelis-Menten constant at which the transport rate is half its maximal value; and $[S]$ is the substrate concentration.

Statistical differences between cell lines or treatments were determined by an unpaired two-tailed Student $t$ test (GraphPad Prism version 7; GraphPad Software Inc.). A $P$ value of less than 0.05 was considered to be statistically significant $(* P<0.05, * * P<0.01$, and $* * * P<0.001)$.

\section{Results}

Effects of PROB on Pharmacokinetics of FSM in Cynomolgus Monkeys. The mean FSM plasma concentration-time profiles after intravenous administration of $2 \mathrm{mg} / \mathrm{kg}$ of FSM in the absence and presence of PROB ( $40 \mathrm{mg} / \mathrm{kg}$, i.v.) are illustrated in Fig. 2A. The plasma concentrations of FSM were higher in the presence than in the absence of PROB at all time points and in all animals. This statement was supported

TABLE 1

Pharmacokinetic parameters of FSM and PROB in cynomolgus monkeys $(n=3)$ following intravenous administration of PROB alone $(40 \mathrm{mg} / \mathrm{kg})$, FSM alone $(2 \mathrm{mg} / \mathrm{kg})$, and PROB with FSM

Pharmacokinetic parameters were determined as described in Materials and Methods. Data are shown as mean \pm S.D. $(\mathrm{n}=3)$. PROB was intravenously dosed 30 minutes prior to FSM administration in the coadministration treatment group.

\begin{tabular}{|c|c|c|c|c|}
\hline Analyte/Parameter & PROB Alone & FSM Alone & FSM with PROB & Ratio $(90 \% \mathrm{CI})$ \\
\hline \multicolumn{5}{|l|}{ FSM } \\
\hline $\mathrm{AUC}_{0-24 \mathrm{~h}}(\mu \mathrm{M} \cdot \mathrm{h})$ & NA & $11.5 \pm 0.7$ & $47.7 \pm 5.9 * *$ & $4.1(3.6-4.8)$ \\
\hline $\mathrm{CL}_{\mathrm{TOT}}(\mathrm{ml} / \mathrm{min}$ per kilogram $)$ & NA & $8.8 \pm 0.6$ & $2.1 \pm 0.3 * * *$ & $0.24(0.21-0.28)$ \\
\hline $\mathrm{Vd}_{\mathrm{SS}}(1 / \mathrm{kg})$ & NA & $0.33 \pm 0.11$ & $0.21 \pm 0.03$ & $0.66(0.31-1.4)$ \\
\hline$T_{1 / 2}(\mathrm{~h})$ & NA & $5.9 \pm 1.4$ & $4.7 \pm 0.8$ & $0.82(0.40-1.7)$ \\
\hline $\mathrm{CL}_{\mathrm{R}}(\mathrm{ml} / \mathrm{min}$ per kilogram $)$ & NA & $3.7 \pm 0.6$ & $0.44 \pm 0.12 * *$ & $0.12(0.11-0.13)$ \\
\hline $\mathrm{CL}_{\mathrm{NR}}(\mathrm{ml} / \mathrm{min}$ per kilogram $)$ & NA & $5.0 \pm 0.6$ & $1.7 \pm 0.2 * *$ & $0.34(0.27-0.42)$ \\
\hline $\mathrm{ER}_{\mathrm{R}}$ & NA & $49.5 \pm 8.8$ & $5.9 \pm 1.4^{* *}$ & $0.12(0.11-0.13)$ \\
\hline \multicolumn{5}{|l|}{ PROB } \\
\hline $\mathrm{AUC}_{0-24 \mathrm{~h}}(\mu \mathrm{M} \cdot \mathrm{h})$ & $3436 \pm 655$ & NA & $3386 \pm 860$ & $0.98(0.58-1.6)$ \\
\hline $\mathrm{C}_{24 \mathrm{~h}}(\mu \mathrm{M})$ & $7.3 \pm 5.7$ & NA & $8.9 \pm 6.5$ & $1.1(0.37-3.2)$ \\
\hline $\mathrm{CL}_{\mathrm{TOT}}(\mathrm{ml} / \mathrm{min}$ per kilogram $)$ & $0.69 \pm 0.12$ & NA & $0.71 \pm 0.18$ & $1.0(0.60-1.7)$ \\
\hline $\mathrm{Vd}_{\mathrm{SS}}(1 / \mathrm{kg})$ & $0.20 \pm 0.01$ & NA & $0.21 \pm 0.02$ & $1.0(0.85-1.3)$ \\
\hline$T_{1 / 2}(\mathrm{~h})$ & $3.7 \pm 0.8$ & NA & $3.7 \pm 0.9$ & $1.0(0.87-1.1)$ \\
\hline
\end{tabular}

CI, confidence interval; $\mathrm{ER}_{\mathrm{R}}$, renal extraction ratio; $\mathrm{NA}$, not applicable; $T_{1 / 2}$, terminal elimination half-life

$* * P<0.01$ and $* * * P<0.001$, when the parameter was compared with that administered with FSM or PROB alone. 
by the significant increase in $\mathrm{AUC}_{0-24 \mathrm{~h}}(11.5 \pm 0.7$ and $47.7 \pm 5.0 \mu \mathrm{M} \cdot \mathrm{h}$ after intravenous administration of $2 \mathrm{mg} / \mathrm{kg}$ FSM alone and with $40 \mathrm{mg} / \mathrm{kg}$ PROB, respectively; $P<0.01$ ) (Table 1). The geometric mean and 90\% confidence interval of the FSM $\mathrm{AUC}_{0-24} \mathrm{~h}$ ratio was 4.1 (3.6-4.8). Consistently, the $\mathrm{CL}_{\mathrm{TOT}}$ of FSM was reduced significantly by PROB pretreatment ( $8.8 \pm 0.6$ vs. $2.1 \pm 0.3 \mathrm{ml} / \mathrm{min}$ per kilogram; $P<0.001$ ). However, the administration of PROB did not cause a significant difference in $\mathrm{Vd}_{\mathrm{SS}}$ and terminal elimination half-life of FSM although the mean values were decreased by PROB pretreatment $(0.33 \pm 0.11$ vs. $0.21 \pm 0.031 / \mathrm{kg}$ and $5.9 \pm 1.4$ vs. $4.7 \pm 0.8$ hour, respectively; $P>0.05$ ) (Table 1 ).

The mean urinary excretion rates of FSM in cynomolgus monkeys are shown in Fig. 2C. A 2.1- to 2.4-fold reduction of FSM urinary excretion in the presence of PROB was observed $(P<0.01)$. The $\mathrm{CL}_{\mathrm{R}}$ and extraction ratio of FSM were reduced significantly with PROB pretreatment $(3.7 \pm$ 0.6 vs. $0.44 \pm 0.12 \mathrm{ml} / \mathrm{min}$ per kilogram and $49.5 \pm 8.8$ vs. $5.9 \pm 1.4$, respectively; $P<0.01$ ). Furthermore, the $\mathrm{CL}_{\mathrm{NR}}$ of FSM in the presence of PROB was also reduced markedly $(5.0 \pm 0.6$ vs. $1.7 \pm 0.2 \mathrm{ml} / \mathrm{min}$ per kilogram, respectively; $P<0.01$ ). Therefore, the reduced $\mathrm{CL}_{\text {TOT }}$ by PROB pretreatment was not solely due to either decreased $\mathrm{CL}_{\mathrm{R}}$ or $\mathrm{CL}_{\mathrm{NR}}$.

Figure 2B illustrates the $\mathrm{PROB}$ concentration in plasma after intravenous PROB alone and concurrently with FSM. The PROB plasma concentrations 24 hours after PROB administration $\left(C_{24 \mathrm{~h}}\right)$ were not significantly different $(7.3 \pm 5.7$ and $8.9 \pm 6.5 \mu \mathrm{M}, P>0.05)$ (Table 1). Comparing other PROB pharmacokinetic parameters in the absence or presence of FSM indicates that the mean PROB $\mathrm{AUC}_{0-24 \mathrm{~h}}$,
$C_{24}$ h, $\mathrm{CL}_{\mathrm{TOT}}$, and terminal elimination half-life values were almost identical (Table 1).

Identification of Potential Plasma Endogenous Biomarkers of OAT1 and OAT3 by Metabolomics. To identify potential endogenous plasma probes of OAT1 and OAT3, LC-MS-based metabolomics was used to determine the alterations in plasma concentrations of endogenous compounds between treatments in cynomolgus monkeys. A total of 233 metabolites of known structural identity by matching accurate mass and retention time values with an in-house database of endogenous metabolites were measured, and the concentration values were used to determine statistical significance using the paired Student's $t$ test (Supplemental Table 1). Of those metabolites monitored, 29 endogenous molecules were identified to be present at concentrations at least 3-fold higher at 1 and/or 3 hours in PROB pretreatment groups (administered alone or with FSM) (Table 2), suggesting that the changes of endogenous metabolites in the plasma were associated with inhibition of monkey OAT1 and OAT3, with associated reduction of OAT-mediated renal clearance. However, only some metabolites were able to return to baseline at 24 hours, which were included as a criterion for transporter biomarker candidacy.

Based on the structure similarities, the identified metabolites can be categorized into three subset groups. The first subset of metabolites are long-chain dicarboxylic acids and derivatives (Table 2), which include TDA $\left[\mathrm{HOOC}\left(\mathrm{CH}_{2}\right)_{12} \mathrm{COOH}\right]$ and HDA $\left[\mathrm{HOOC}\left(\mathrm{CH}_{2}\right)_{14} \mathrm{COOH}\right]$. The second subset consists of small acids that are produced by gut microflora.

TABLE 2

Comparison of plasma concentrations of the 29 selected endogenous metabolites in cynomolgus monkeys $(n=3)$ following intravenous administration of PROB alone $(40 \mathrm{mg} / \mathrm{kg})$, FSM alone $(2 \mathrm{mg} / \mathrm{kg})$, and PROB with FSM

Plasma concentrations of approximately 230 endogenous metabolites were determined by liquid chromatography-mass spectrometry (Supplemental Table 1). Each determination was made in samples collected at 1, 3, and 24 hours postdose from three cynomolgus monkeys, and values for compounds with $\geq 3.0$-fold different plasma concentration at any of three time points in the monkeys treated with PROB and FSM are presented. The P values were determined by paired Student's t test (Supplemental Table 1).

\begin{tabular}{|c|c|c|c|c|c|c|}
\hline \multirow{2}{*}{ Metabolite Type and Name } & \multicolumn{3}{|c|}{ Ratio of PROB Alone to FSM Alone } & \multicolumn{3}{|c|}{ Ratio of PROB with FSM to FSM Alone } \\
\hline & $C_{1 \mathrm{~h}}$ & $C_{3 \mathrm{~h}}$ & $C_{24 \mathrm{~h}}$ & $C_{1 \mathrm{~h}}$ & $C_{3 \mathrm{~h}}$ & $C_{24 \mathrm{~h}}$ \\
\hline \multicolumn{7}{|l|}{ Dicarboxylic acids and derivatives } \\
\hline C18-di-OH dihydroxyoctadecanoic acid & 5.1 & 7.6 & 2.1 & 11.7 & 9.5 & 2.5 \\
\hline C18-2 dicarboxylic acid & 3.2 & 3.7 & 0.8 & 8.6 & 5.1 & 0.8 \\
\hline C20-4 dicarboxylic acid & 3.0 & 3.6 & 0.7 & 8.8 & 5.5 & 0.5 \\
\hline C18-1 dicarboxylic acid & 2.7 & 3.6 & 0.7 & 7.8 & 5.8 & 0.5 \\
\hline C16-1 dicarboxylic acid & 2.4 & 2.0 & 0.6 & 4.6 & 2.8 & 0.8 \\
\hline TDA & 2.3 & 3.7 & 2.6 & 8.8 & 13.7 & 2.0 \\
\hline C12 dicarboxylic acid & 2.1 & 3.7 & 1.2 & 1.8 & 2.4 & 2.0 \\
\hline C10-1 dicarboxylic acid & 2.1 & 2.8 & 0.7 & 3.4 & 4.5 & 0.7 \\
\hline C12-1 dicarboxylic acid & 2.1 & 1.2 & 0.9 & 3.1 & 1.7 & 0.8 \\
\hline C12-OH hydroxydidecanoic acid & 2.0 & 1.3 & 1.3 & 3.5 & 2.6 & 1.4 \\
\hline C14-1 dicarboxylic acid & 1.9 & 2.3 & 1.6 & 4.2 & 4.4 & 1.7 \\
\hline Unsaturated tetradecanoyl carnitine (C14-1) & 3.9 & 1.0 & 0.5 & 2.8 & 1.5 & 0.5 \\
\hline Unsaturated lauryl carnitine (C12-1) & 3.3 & 1.1 & 0.6 & 1.9 & 1.2 & 0.6 \\
\hline Lauryl carnitine (C12) & 3.0 & 1.1 & 0.5 & 1.7 & 0.9 & 0.5 \\
\hline \multicolumn{7}{|l|}{ Small acids produced by gut microflora } \\
\hline Indole-3 acetic acid & 8.6 & 11.2 & 1.6 & 7.6 & 7.7 & 1.7 \\
\hline Cresol sulfate & 3.9 & 5.6 & 2.3 & 4.9 & 7.1 & 2.6 \\
\hline Phenyl sulfate & 3.1 & 2.9 & 1.5 & 2.6 & 3.4 & 1.6 \\
\hline Phenyllactic acid & 2.9 & 3.3 & 2.2 & 3.3 & 3.0 & 2.0 \\
\hline Indoxyl sulfate & 2.8 & 2.8 & 1.0 & 6.4 & 7.5 & 1.2 \\
\hline Phenylacetylglycine & 2.6 & 3.3 & 0.5 & 3.4 & 3.4 & 0.6 \\
\hline \multicolumn{7}{|l|}{ Amino acids and derivatives } \\
\hline Hydroxy-isovaleric acid & 3.9 & 1.5 & 4.4 & 12.6 & 1.8 & 6.2 \\
\hline Tyrosine & 3.1 & 2.6 & 1.5 & 2.5 & 1.6 & 0.4 \\
\hline Aspartic acid & 2.2 & 3.0 & 2.2 & 1.6 & 2.5 & 0.5 \\
\hline \multicolumn{7}{|l|}{ Others } \\
\hline PDA & 4.5 & 4.8 & 1.4 & 5.6 & 6.0 & 1.5 \\
\hline HVA & 4.1 & 3.2 & 0.8 & 4.6 & 4.0 & 1.3 \\
\hline Glucuronic acid & 8.8 & 8.5 & 2.6 & 10.5 & 11.2 & 2.4 \\
\hline Pantothenic acid & 2.8 & 1.1 & 0.8 & 3.0 & 1.7 & 0.9 \\
\hline Xanthurenic acid & 3.8 & 5.2 & 0.4 & 7.4 & 8.1 & 0.7 \\
\hline Kynurenic acid & 3.5 & 4.1 & 0.6 & 6.6 & 6.0 & 1.0 \\
\hline
\end{tabular}


The metabolites include indole-3 acetic acid, cresol sulfate, phenyl sulfate, phenyllactic acid, indoxyl sulfate, and phenylacetylglycine. The third are amino acids and derivatives, which include hydroxy isovaleric acid, tyrosine, and aspartic acid. The metabolites whose plasma concentrations were significantly increased by PROB pretreatments include PDA, HVA, glucuronic acid, pantothenic acid, xanthurenic acid, and kynurenic acid (Table 2). The administration of PROB caused increases in plasma PDA and HVA concentrations at 1 and 3 hours (approximately 3 - to 6-fold), and the concentrations returned to baseline at 24 hours (Table 2). PDA and HVA represent novel types of endogenous biomarkers not known previously to interact with monkey and human OAT1 and OAT3. Therefore, we conducted follow-up experiments to determine the time course of change in plasma PDA and HVA concentrations and transporterexpressing cell uptake to comprehend the sensitivity and specificity of the probes as described subsequently.

Time-Dependent Effects on Plasma PDA, HVA, HDA, and TDA Levels of PROB Pretreatment. Administration of $40 \mathrm{mg} / \mathrm{kg}$ PROB intravenously caused increases in plasma PDA and HVA concentrations in monkeys. The plasma PDA concentration increased by approximately 3 -fold over the first 24 hours $(113.5 \pm 23.3$ and $120.0 \pm 17.9$ vs. $40.5 \pm 2.2 \mu \mathrm{M} \cdot \mathrm{h}, P<0.05)$, and gradually declined to the basal level $(1.5 \pm 0.3 \mu \mathrm{M})$ at 24 hours after PROB pretreatment (Fig. 3A; Table 3). The PDA concentrations were greater at any time points after PROB pretreatment compared with those after FSM administration. Similarly, the increase in plasma HVA concentration was persistent. The plasma HVA concentration peaked at 3-4 hours, returning to the baseline $(85 \pm 32 \mathrm{nM})$ within 24 hours after PROB pretreatment with approximately 2-fold increase in AUC $(6.5 \pm 1.0$ and $8.7 \pm 1.9$ vs. $4.1 \pm 0.7 \mu \mathrm{M} \cdot \mathrm{h}, P<0.05$ ) (Fig. $3 \mathrm{~B}$; Table 3 ). As shown in Table 3, PROB pretreatment significantly decreased $\mathrm{CL}_{\mathrm{R}}$ of PDA compared with FSM treatment $(P<0.05)$. The pretreatment also decreased the $\mathrm{CL}_{\mathrm{R}}$ value of HVA, although the reduction was not statistically significant.

We also detected the effects of PROB on plasma concentrations of TDA and HDA since they are reported as substrates of OAT1 and OAT3. PROB pretreatment increased plasma concentrations of TDA and other long-chain dicarboxylic acids in monkeys (Table 2). Figure 3, C and D show that PROB pretreatments increase plasma concentrations, although the increases were not statistically significant (Table 3).

Measurement of PDA and HVA Uptake in Stable Cell Lines Constitutively Expressing Renal Transporter. To determine whether PDA and HVA are substrates for major renal drug transporters, the cellular uptake of the molecules was measured at a single concentration (1 and $5 \mu \mathrm{M}$ for PDA and HVA, respectively) in human OAT1-, OAT2-, OAT3-, OAT4-, OCT2-, MATE1-, MATE2K-, OATP1B1, OATP1B3-, and NTCP-HEK cells after 5-minute incubation. The cellular uptake of PDA and HVA into the HEK cells stably transfected with the cynomolgus monkey renal OATs was not evaluated because in vitro models are not available in the United States (Tahara et al., 2005). The uptake of PDA in OAT1- and OAT3-HEK cells was approximately 55and 52-fold higher than that in the control cells (1757 \pm 703 vs. $30.6 \pm$ 6.7 and $1548 \pm 40.0$ vs. $29.8 \pm 5.9 \mathrm{pmol} / \mathrm{mg}$ after 5 minutes, respectively) (Fig. 4A). In addition, the PDA uptake in OAT1- and OAT3-HEK cells is significantly inhibited by PROB, a potent OAT1 and OAT3 inhibitor (Fig. 4A). Furthermore, PDA is a substrate for human OAT4 because the uptake of PDA in OAT4-HEK cells is significantly greater than that in Mock-HEK cells $(1.8$-fold; $P<0.01)$ and the uptake is reduced by $1 \mathrm{mM}$ PROB (Fig. 5A). On the other hand, there was no significant OAT2-, OCT2-, MATE1-, and MATE2Kmediated uptake of PDA compared with the control $(P>0.05)$ (Figs. 4A and 5A). Similarly, the uptake of HVA in OAT1- and OAT3-expressing cells was approximately 45- and 6-fold higher than those in the control cells $(8.33 \pm 0.40$ vs. $0.18 \pm 0.02$ and $1.09 \pm 0.09$ vs. $0.18 \pm 0.02$ $\mathrm{pmol} / \mathrm{mg}$ after 5 minutes, respectively; $P<0.001$ ) (Fig. 4B). Additionally, there was also significant OAT2-mediated uptake of HVA compared with the control (5-fold; $P<0.001)$. However, there was no significant OCT2-, MATE1-, and MATE2K-mediated uptake of HVA compared with the control $(P>0.05)$. Moreover, both PDA and HVA are not substrates for human hepatic transporters OATP1B1, OATP1B3, and NTCP because there are no significant differences in the uptake between the transporter-overexpressing cells and mock cells $(P>0.05)$ (Supplemental Fig. 2).
A

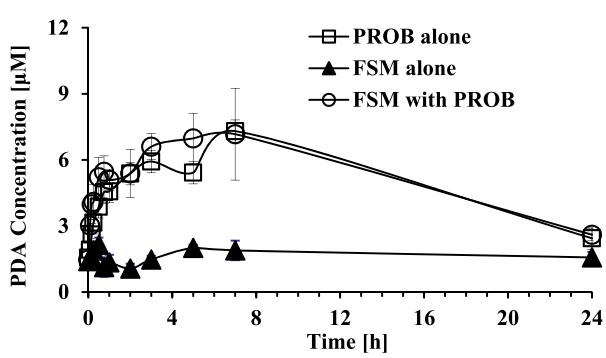

C

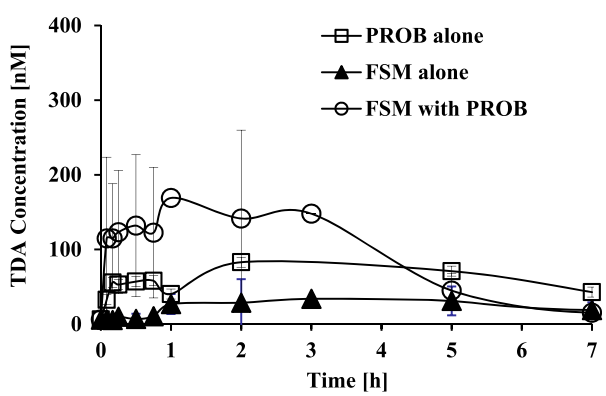

B

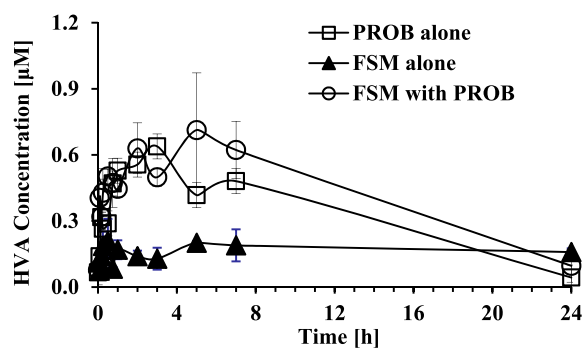

D

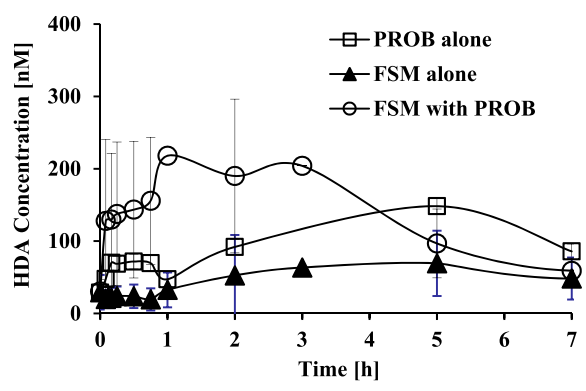

Fig. 3. Effect of $P R O B$ on plasma concentration-time curves of PDA (A), HVA (B), TDA (C), and HDA (D) in three cynomolgus monkeys after intravenous administration of PROB alone (40 mg/kg; open squares), FSM alone (2 mg/kg, close triangles), and PROB with FSM (open circles). 
TABLE 3

Pharmacokinetic parameters of PDA, HVA, TDA, and HDA in three cynomolgus monkeys following intravenous administration of PROB alone $(40 \mathrm{mg} / \mathrm{kg})$, FSM alone $(2 \mathrm{mg} / \mathrm{kg})$, and PROB with FSM

Pharmacokinetic parameters were determined as described in Materials and Methods. Data are shown as mean \pm S.D. $(\mathrm{n}=3)$. PROB was intravenously dosed 30 minutes prior to FSM administration in the coadministration treatment group.

\begin{tabular}{|c|c|c|c|c|c|}
\hline \multirow{2}{*}{ Analyte/Parameter } & \multicolumn{2}{|c|}{ PROB Alone } & \multirow{2}{*}{$\frac{\text { FSM Alone }}{\text { Value }}$} & \multicolumn{2}{|c|}{ FSM with PROB } \\
\hline & Value & Ratio $(90 \% \mathrm{CI})^{a}$ & & Value & Ratio $(90 \% \mathrm{CI})^{a}$ \\
\hline \multicolumn{6}{|l|}{ PDA } \\
\hline $\mathrm{AUC}_{0-24 \mathrm{~h}}(\mu \mathrm{M} \cdot \mathrm{h})$ & $113.5 \pm 23.3^{*}$ & $2.8(2.0-3.9)$ & $40.5 \pm 2.2$ & $120.0 \pm 17.9 * *$ & $2.9(2.1-4.1)$ \\
\hline$C_{\max }(\mu \mathrm{M})$ & $7.3 \pm 1.2 *$ & $3.1(2.2-4.3)$ & $2.4 \pm 0.2$ & $7.9 \pm 1.1 * *$ & $3.3(2.3-4.8)$ \\
\hline $\mathrm{CL}_{\mathrm{R}}(\mathrm{ml} / \mathrm{min}$ per kilogram) & $0.4 \pm 0.2 *$ & $0.10(0.01-1.6)$ & $4.9 \pm 3.3$ & $2.7(2.2 \text { and } 3.1)^{b}, * *$ & $0.39(0.33 \text { and } 0.46)^{b}$ \\
\hline \multicolumn{6}{|l|}{ HVA } \\
\hline $\mathrm{AUC}_{0-24 \mathrm{~h}}(\mu \mathrm{M} \cdot \mathrm{h})$ & $6.5 \pm 1.0 *$ & $1.6(1.1-2.3)$ & $4.1 \pm 0.7$ & $8.7 \pm 1.9 *$ & $2.1(1.9-2.4)$ \\
\hline$C_{\max }(\mu \mathrm{M})$ & $0.68 \pm 0.03 * *$ & $2.8(2.0-3.8)$ & $0.25 \pm 0.04$ & $0.78 \pm 0.22 *$ & $3.1(2.1-4.6)$ \\
\hline $\mathrm{CL}_{\mathrm{R}}(\mathrm{ml} / \mathrm{min}$ per kilogram$)$ & $1.3 \pm 0.5$ & $0.8(0.4-1.6)$ & $1.6 \pm 0.2$ & $0.9(0.9 \text { and } 0.8)^{b}, *$ & $0.50(0.49 \text { and } 0.51)^{b}$ \\
\hline \multicolumn{6}{|l|}{ TDA } \\
\hline $\mathrm{AUC}_{0-7 \mathrm{~h}}(\mathrm{nM} \cdot \mathrm{h})$ & $449 \pm 233^{*}$ & $2.8(1.6-5.0)$ & $168 \pm 121$ & $526 \pm 250$ & $3.3(1.1-10.3)$ \\
\hline$C_{\max }(\mathrm{nM})$ & $97 \pm 43^{*}$ & $2.3(1.8-3.0)$ & $42 \pm 21$ & $195 \pm 110$ & $4.3(1.1-17.7)$ \\
\hline \multicolumn{6}{|l|}{ HDA } \\
\hline $\mathrm{AUC}_{0-7 \mathrm{~h}}(\mathrm{nM} \cdot \mathrm{h})$ & $592 \pm 224^{*}$ & $1.9(0.9-3.7)$ & $355 \pm 263$ & $870 \pm 321$ & $2.7(0.9-8.0)$ \\
\hline$C_{\max }(\mathrm{nM})$ & $148 \pm 109$ & $2.0(1.1-3.9)$ & $69 \pm 45$ & $223 \pm 107 *$ & $3.3(1.7-6.6)$ \\
\hline
\end{tabular}

CI, confidence interval.

${ }^{a}$ Ratio of pharmacokinetics parameter of PROB treatment to FSM treatment.

${ }^{b}$ Cage malfunction prevented urine collection from one monkey in the coadministration treatment group $(n=2)$.

$* P<0.05$ and $* * P<0.01$, when the parameter was compared with that administered with FSM alone.

The affinities of OAT1- and OAT3-meditated transport for PDA and HVA were further determined over a range of PDA and HVA concentrations $(0.2-500 \mu \mathrm{M})$ in OAT1- and OAT3-HEK cells after 2.5-minute incubation. The incubation time was set by the linear-uptake condition and the lower limit of bioanalytical methods (Supplemental Fig. 1). As shown in Fig. 6, the apparent $K_{\mathrm{m}}$ values were $33.0 \pm 5.3$ and $52.1 \pm 15.3 \mu \mathrm{M}$ for OAT1- and OAT3-mediated uptake of PDA, respectively, while the apparent $K_{\mathrm{m}}$ values of OAT1-, OAT-2, and OAT3-mediated uptake of HVA were $108 \pm 6.2$, $124 \pm 10.8$, and $438 \pm 63 \mu \mathrm{M}$, respectively.

\section{Discussion}

OAT transporters are mainly expressed in RPTCs and mediate active renal secretion of their substrates. Inhibition of OAT transporter function could lead to decreased renal elimination and increased plasma exposure of xenobiotics and endogenous metabolites that are transported by these transporters. The general approach of using cynomolgus monkey as a model for study of human transporter function has become routine and takes advantage of the similarity and function of drug transporters between humans and monkeys (Tahara et al., 2006; Shen et al., 2013, 2016b). It is recognized that such approaches can identify endogenous biomarker candidates that are selective for a specific transporter (Chu et al., 2015; Shen et al., 2016a; Thakare et al., 2017). To demonstrate that cynomolgus monkey permits quantitative prediction of human OATmediated DDIs, we measured the effects of the clinically relevant OAT inhibitor PROB on the pharmacokinetics of the OAT1 and OAT3 probe substrate FSM in monkeys, and compared these results with the changes observed in human subjects. As shown in Fig. 2, the intravenous
A

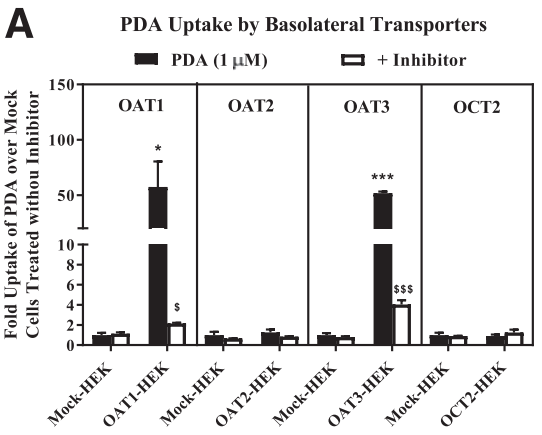

B HVA Uptake by Basolateral Transporter

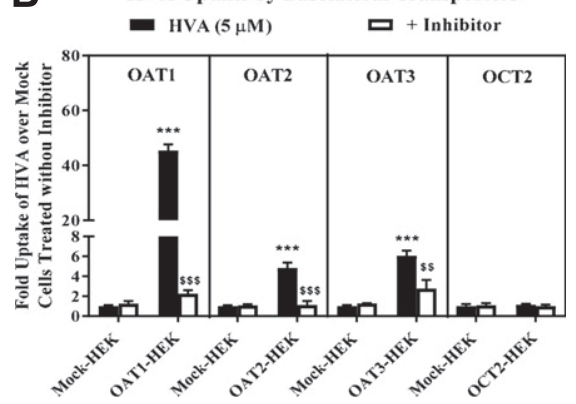

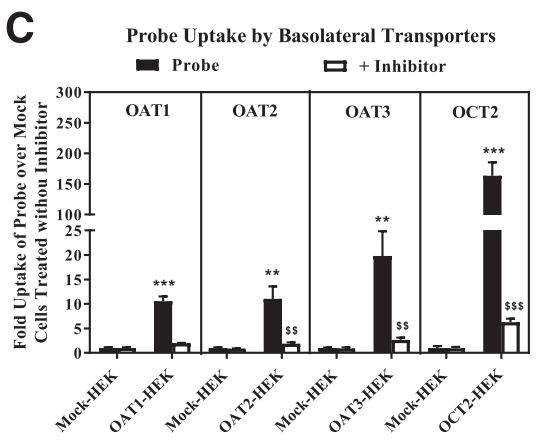

Fig. 4. Profiling of the transport of PDA and HVA by major drug transporters expressed at the basolateral membrane of RPTC. Uptake in the HEK cells stably transfected with the control vector (Mock-HEK), OAT1, OAT2, OAT3, or OCT2 was measured after 5-minute incubation at $37^{\circ} \mathrm{C}$ with PDA $(1 \mu \mathrm{M})(\mathrm{A})$, HVA $(5 \mu \mathrm{M})(\mathrm{B})$, and radiolabeled probe substrates [1 $\mu \mathrm{M}\left[{ }^{3} \mathrm{H}\right]$ para-aminohippuric acid (OAT1), $1 \mu \mathrm{M}\left[{ }^{3} \mathrm{H}\right]$ penciclovir (OAT2), $1 \mu \mathrm{M}\left[{ }^{3} \mathrm{H}\right]-$ estrone-3-sulfate (OAT3), and $2 \mu \mathrm{M}\left[{ }^{14} \mathrm{C}\right]$ metformin (OAT2)] (C). Incubations were conducted in the absence and presence of $1 \mathrm{mM}$ PROB (OAT1 and OAT3), $100 \mu \mathrm{M}$ indomethacin (OAT2), or $100 \mu \mathrm{M}$ pyrimethanmine (OCT2) to evaluate the effects of these inhibitors on PDA and HVA uptake. Each value represents the mean \pm S.D. $(n=3) . * P<0.05$; $* * P<0.01 ; * * * P<0.001$ statistically significantly different from uptake in MockHEK cells, and ${ }^{\$} P<0.05 ;{ }^{\$ \$} P<0.01 ;{ }^{\$ \$} P<$ 0.001 statistically significantly different from uptake in the absence of an inhibitor. 

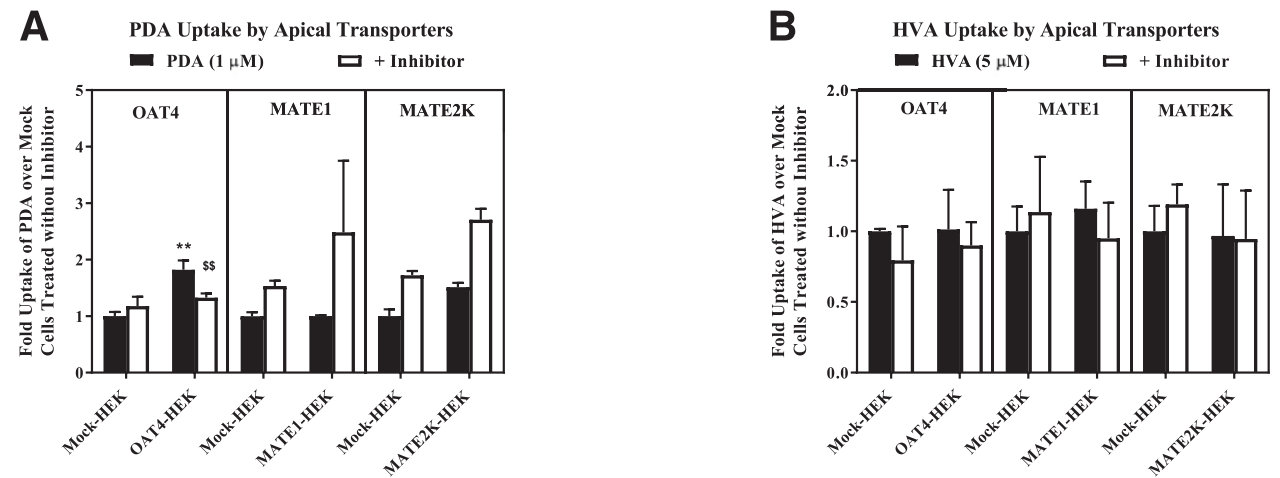

Fig. 5. Profiling of the transport of PDA and HVA by major drug transporters expressed at the apical membrane of RPTC. Uptake in the HEK cells stably transfected with the control vector (Mock-HEK), OAT4, MATE1, or MATE2K was measured after 5-minute incubation at $37^{\circ} \mathrm{C}$ with PDA $(1 \mu \mathrm{M})(\mathrm{A}), \mathrm{HVA}$ $(5 \mu \mathrm{M})(\mathrm{B})$, and $\left[{ }^{3} \mathrm{H}\right]$ estrone-3-sulfate $(1 \mu \mathrm{M})$ and $\left[{ }^{14} \mathrm{C}\right]$ metformin $(2 \mu \mathrm{M})(\mathrm{C})$. Incubations

C Probe Uptake by Apical Transporters

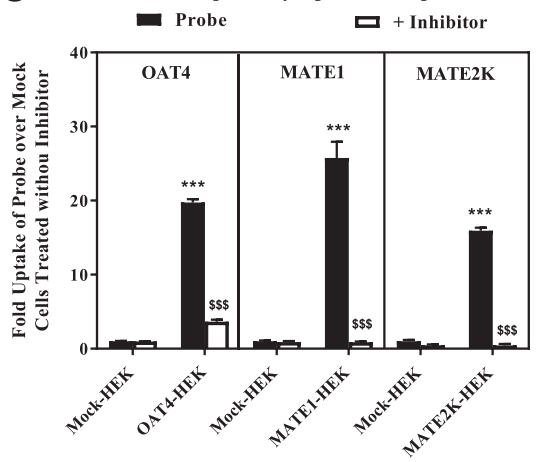

are conducted in the absence and presence of $1 \mathrm{mM}$ PROB (OAT4) or $100 \mu \mathrm{M}$ pyrimethanmine (MATE1 and MATE2K) to evaluate the effects of these inhibitors on PDA and HVA uptake. Each value represents the mean \pm S.D. $(n=3)$. *** $P<0.001$ statistically significantly different from uptake in Mock-HEK cells, and ${ }^{\$}$ ${ }^{\$} P<0.001$ statistically significantly different from uptake in the absence of an inhibitor. administration of PROB generated a plasma total $C_{24 \mathrm{~h}}$ of $7.3 \pm 5.7 \mu \mathrm{M}$ (Fig. 2; Table 1). Based on the $\mathrm{IC}_{50}$ values for both monkey OAT1 and OAT3 $(<10 \mu \mathrm{M})$ (Tahara et al., 2005), sustained inhibition of OAT1 and OAT3 transport function is anticipated. In agreement with the in vitro inhibition, a pronounced increase in AUC of FSM, a known substrate of OAT1 and OAT3, resulting from PROB pretreatment was evident (Fig. 2; Table 1). At the relevant dose (i.e., $40 \mathrm{mg} / \mathrm{kg}$, i.v.), PROB decreased the FSM $C_{R}$ value by 8.3 -fold in cynomolgus monkeys. The decreases in the $\mathrm{CL}_{\mathrm{R}}$ values of coadministered FSM observed in the clinic were 3.6- to 5.1-fold for PROB (Chennavasin et al., 1979; Smith et al., 1980). Furthermore, PROB decreased FSM $\mathrm{CL}_{\mathrm{NR}}$ in monkeys and humans by 2.9 - and 1.5 -fold, respectively, suggesting inhibition of FSM metabolism in both species. In the current study, we demonstrated that intravenous pretreatment of cynomolgus monkeys with PROB $(40 \mathrm{mg} / \mathrm{kg})$ increased in the AUC of FSM by 4.1fold, which is consistent with the values (3.1- to 3.7-fold) reported in humans and indicates that DDIs associated with OAT transporter inhibitions could be reproduced in cynomoglous monkey. These results support previous findings in cynomolgus monkeys where it was found that OAT1 and OAT3 exhibit very similar transport kinetics compared with corresponding human orthologs, and cynomolgus monkeys are recommended as more appropriate alternative systems for predicting DDIs involving renal drug transporters in humans (Tahara et al., 2005, 2006).

In the present study, we applied a combination of fit-for-purpose untargeted metabolomics and quantitative multiple reactions monitoring LC-MS/MS methods (Fig. 1) to determine if OAT inhibition is associated with changes in plasma concentrations of endogenous metabolites that are reflective of OAT function. Of the 233 plasma metabolites examined using the metabolomics method, 29 metabolites including PDA and HVA were significantly increased at 1 or 3 hours in plasma from monkeys by PROB. Of those, a number of metabolites identified had not previously been reported as substrates for OAT1 and
OAT3 (Table 2). LC-MS/MS methods were further optimized for determination of plasma PDA and HVA concentration-time profiles. We found PDA and HVA to be consistently elevated in plasma from cynomolgus monkeys pretreated with PROB alone and with FSM ( $\mathrm{AUC}_{0-24 \mathrm{~h}}$ of 2.8- to 2.9-fold and 1.6- to 2.1-fold, respectively), similar to the increase in plasma FSM concentration (4.1-fold) (Figs. 2 and 3; Tables 1 and 3). Furthermore, we demonstrated that PDA and HVA are substrates for human OAT1, OAT3, OAT2 (HVA), and OAT4 (PDA) but not OCT2, MATE1, MATE2K, OATP1B1, OATP1B3, and NTCP (Figs. 4 and 5; Supplemental Fig. 2). However, we did not study the transport of PDA and HVA by cynomolgus monkey OAT1 and OAT3 because in vitro cell models are not available in the United States. The species-dependent differences in OAT1- and OAT3-mediated transport of PDA and HVA cannot be excluded, although monkey OAT1 and OAT3 exhibit similar transport kinetics (i.e., $K_{\mathrm{m}}$ and $V_{\max }$ values) to human orthologs (Tahara et al., 2005). Collectively, the results from the present study demonstrate that circulating PDA and HVA can be potentially used as endogenous biomarkers of OAT1 and OAT3 inhibition.

A few years ago, an investigation was conducted examining the changes of plasma and urine metabolites with murine Oat1 (Slc22a6) deficiency. Using untargeted metabolomics analysis, the researchers identified several physiologically important metabolites, including PDA, that were increased in the plasma from Oat1 knockout mice compared with wild-type animals, suggesting they are substrates for mouse Oat1 (Wikoff et al., 2011). In agreement, PROB increased the plasma HVA levels in rhesus monkey (Bacopoulos et al., 1978). PDA has been shown to undergo active renal secretion in humans, and elevated plasma PDA was observed in patients with renal insufficiency (Coburn et al., 2002). It is worth noting that the present study shows a considerable variation in the urinary excretion and $\mathrm{CL}_{\mathrm{R}}$ of PDA and HVA (Fig. 3, B and D; Table 3). In addition, the volumes of urine samples collected in PROB groups during normal diuresis and 


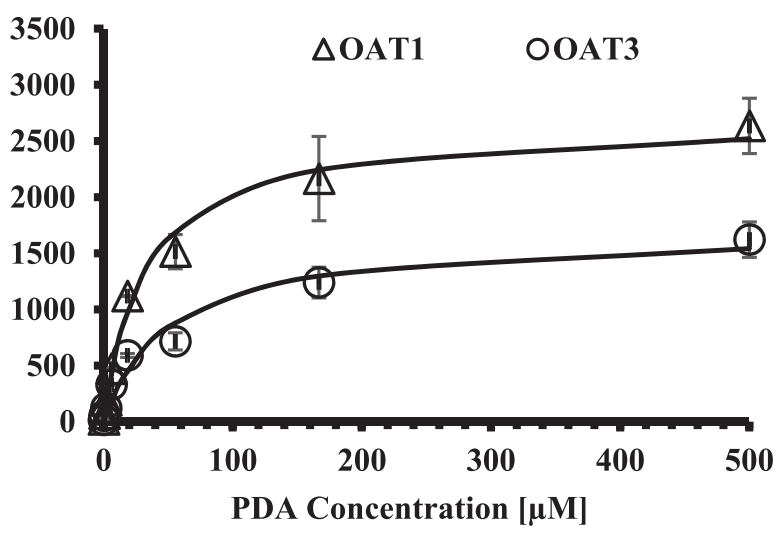

B

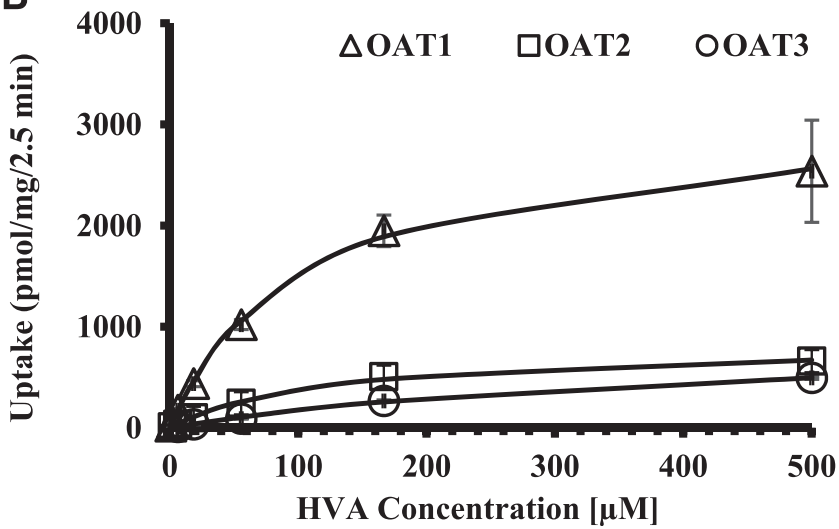

Fig. 6. Concentration-dependent uptake of PDA (A) and HVA (B) by human OAT1 and OAT3. Cells were incubated with PDA or HVA $(0.23-500 \mu \mathrm{M})$ for 2 minutes (linear range). The net OAT1- (open triangle) or OAT3-mediated uptake (open circle) was calculated by subtracting that in mock cells from that in OAT1- or OAT3-HEK cells. The curves represent the best fit of the Michaelis-Menten equation. Each value represents the mean \pm S.D. $(n=3)$.

forced diuresis (i.e., administration of PROB alone and with FSM) are significantly different. Despite large variation, PROB pretreatments, either alone or with FSM, significantly reduced the $C L_{R}$ values of PDA in monkeys (ratios of 0.10 and $0.33-0.46$, respectively), similar to the decrease in FSM $\mathrm{CL}_{\mathrm{R}}[0.12(0.11-0.13)]$ (Tables 1 and 3).

Although the plasma levels of HVA are significantly increased by PROB pretreatments compared with FSM treatment, the $\mathrm{CL}_{\mathrm{R}}$ value was only slightly reduced by PROB pretreatments (ratios of 0.8 and 0.49 0.50 , respectively) and the changes were not statistically significant. It is unclear why PROB pretreatments decreased the $\mathrm{CL}_{\mathrm{R}}$ of $\mathrm{HVA}$ to a lesser extent compared with PDA. However, the following observations may shed some light. Based on the in vitro uptake experiments, the renal basolateral transporters OAT1, OAT2, and OAT3 are involved in the uptake of HVA while PDA is only transported by OAT1 and OAT3 (Figs. 4 and 6). OAT2 is less sensitive to the inhibition by PROB compared with OAT1 and OAT3 ( $\mathrm{IC}_{50}$ of 393-766 $\mu \mathrm{M}$ vs. $<10 \mu \mathrm{M}$ ) (Enomoto et al., 2002; Jia et al., 2015). In contrast to our findings, previous studies examining expression of human OATs in the choroid plexus and their interactions with neurotransmitter metabolites have reported that HVA is not a substrate for human OATs (OAT1 and OAT3) in mouse cells (second segment of the proximal tubule cells, S2 cells) stably expressing OAT1 or OAT3 (Alebouyeh et al., 2003); the difference in transport profiling is probably due to the use of different host cells (HEK 293 cells vs. S2 cells), and endogenous expression of mouse Oat1, Oat2, and Oat3 in S2 host cells would mask HVA uptake. Although we showed high-affinity uptake of PDA and HVA by basolateral transporters OAT1, OAT3, and/or OAT2 into stably transfected HEK cells (Figs. 4 and 6), the mechanism(s) of the export of PDA and HVA out of the proximal tubule cells into the urine is not fully understood. These molecules may be extruded out of the cell by the efflux transporters MRP2 and MRP4 since most MRP2 and MRP4 substrates are organic anions, which are localized in the apical membrane of RPTCs. In addition, the renal apical transporter OAT4 may play a role in the transport of PDA from RPTCs into the urine since OAT4 can operate in efflux mode in addition to influx fashion (Hagos et al., 2007).

PROB is a selective inhibitor of OAT1 and OAT3 since its $\mathrm{IC}_{50}$ values toward other transporters are greater than $25 \mu \mathrm{M}$ (Supplemental Table 2), although for other monkey transporters there is still paucity of complete data for PROB inhibition. Therefore, even though the results presented herein showcase PDA and HVA as novel cynomolgus monkey plasma OAT biomarkers, it cannot be assumed that the results translate directly to human subjects. In addition, we cannot exclude that the formation of the biomarkers studied may be affected by PROB. Moreover, PDA is the dead-end catabolite of the B6 vitamins including pyridoxine, pyridoxamine, and pyridoxal (Merrill and Henderson, 1990). Although the plasma PDA baseline level exhibits acceptable interindividual variability in monkeys and humans (1.6 $\pm 0.4 \mu \mathrm{M}$ and $43 \pm 17 \mathrm{nM}$ ) (Coburn et al., 2002), the use of vitamin B6 supplements causes elevation of PDA levels far above the normal physiologic level (Zempleni, 1995; Coburn et al., 2002). Therefore, the use of vitamin B6 supplements should be excluded in a clinical study with the goal of assessing a change in PDA plasma level.

Emerging metabolomics and genome-wide association data revealed that HDA and TDA, two fatty acid dicarboxylates, were potential endogenous biomarkers of human OATP1B1 (Yee et al., 2016). Although HDA and TDA are substrates of OATP1B1, OAT1 and OAT3 were also involved in the disposition of HDA and TDA (Yee et al., 2016). Consistently, we have observed that administration of rifampin, a selective inhibitor of hepatic OATPs over renal OATs, increased the AUCs of HDA and TDA by approximately 2-fold in human subjects (Shen et al., 2017). Although these results indicated that HDA and TDA are endogenous biomarkers of OATP1B1, the in vitro transport profiling suggested the involvement of OAT1 and OAT3 in the disposition of HDA and TDA (Yee et al., 2016). Metabolomics analysis in this study demonstrated the significant elevations in plasma levels of at least 20 dicarboxylic acids and fatty acids in the monkeys pretreated with PROB (Supplemental Table 1; Table 2). A similar trend was more evident for TDA with significant increases of AUC in the LC-MS/MS data set (Fig. 3C; Table 3). A comparable degree of increase has been observed with HDA (Fig. 3D; Table 3). As a result, HDA and TDA may serve as dual hepatic OATP and renal OAT biomarkers although further studies are needed for the relevance of clinical interactions. The concentration-time profiles for TDA and HDA appear different in the PROB-alone and with FSM groups although the concentrations in both groups are greater than those in that of FSM alone (Fig. 3, C and D). This is likely due to the additional inhibitory effect of FSM toward OAT1and OAT3-mediated transport of TDA and HDA (Hasannejad et al., 2004; Nieskens et al., 2016).

In conclusion, the use of monkey as a transporter-mediated DDI model along with metabolomics has been demonstrated to be a useful approach for transporter biomarker identification. Our investigations showed that PDA and HVA are novel blood biomarkers of monkey renal OAT since the changes in plasma exposures of PDA and HVA are similar to that of a probe substrate in monkeys. Although interspecies differences in the transport and disposition of PDA and HVA need be considered, the information obtained 
in this monkey study on the extent of these endogenous compounds after pretreatment with PROB along with the results from stably transporteroverexpressing cell lines suggest that PDA and HVA are candidate biomarkers of OAT1 and OAT3 for use in clinical settings.

\section{Acknowledgments}

We thank Joseph Cantone and Dr. Dieter Drexler for supporting the bioanalysis of PDA and HVA in the OAT4, OATP1B1, OATP1B3, and NTCP uptake experiments.

\section{Authorship Contributions}

Participated in research design: Shen, Nelson, Oliveira, Lai, Humphreys.

Conducted experiments: Shen, Oliveira, Zhang, Mcnaney, Gu, Chen, Su.

Contributed new reagents or analytic tools: Shen, Nelson, Oliveira.

Performed data analysis: Shen, Nelson, Oliveira, Zhang, Shipkova, Reily, Gan, Marathe, Humphreys.

Wrote or contributed to the writing of the manuscript: Shen, Nelson, Oliveira, Lai, Marathe, Humphreys.

\section{References}

Alebouyeh M, Takeda M, Onozato ML, Tojo A, Noshiro R, Hasannejad H, Inatomi J, Narikawa S, Huang XL, Khamdang S, et al. (2003) Expression of human organic anion transporters in the choroid plexus and their interactions with neurotransmitter metabolites. Journal of Pharmacological Sciences 93:430-436.

Bacopoulos NG, Heninger GR, and Roth RH (1978) Effects of haloperidol and probenecid on plasma and CSF dopamine metabolites in the rhesus monkey (Macacca mulatta). Life Sci 23: $1805-1812$.

Bergagnini-Kolev MC, Hebert MF, Easterling TR, and Lin YS (2017) Pregnancy increases the renal secretion of $\mathrm{N}(1)$-methylnicotinamide, an endogenous probe for renal cation transporters, in patients prescribed metformin. Drug Metab Dispos 45:325-329.

Chennavasin P, Seiwell R, Brater DC, and Liang WM (1979) Pharmacodynamic analysis of the furosemide-probenecid interaction in man. Kidney Int 16:187-195.

Chu X, Chan GH, and Evers R (2017) Identification of endogenous biomarkers to predict the propensity of drug candidates to cause hepatic or renal transporter-mediated drug-drug interactions. J Pharm Sci 106:2357-2367.

Chu X, Shih SJ, Shaw R, Hentze H, Chan GH, Owens K, Wang S, Cai X, Newton D, Castro-Perez J, et al. (2015) Evaluation of cynomolgus monkeys for the identification of endogenous biomarkers for hepatic transporter inhibition and as a translatable model to predict pharmacokinetic interactions with statins in humans. Drug Metab Dispos 43:851-863.

Coburn SP, Reynolds RD, Mahuren JD, Schaltenbrand WE, Wang Y, Ericson KL, Whyte MP, Zubovic YM, Ziegler PJ, Costill DL, et al. (2002) Elevated plasma 4-pyridoxic acid in rena insufficiency. Am J Clin Nutr 75:57-64.

Davies B and Morris T (1993) Physiological parameters in laboratory animals and humans. Pharm Res 10:1093-1095.

Enomoto A, Takeda M, Shimoda M, Narikawa S, Kobayashi Y, Kobayashi Y, Yamamoto T, Sekine T, Cha SH, Niwa T, et al. (2002) Interaction of human organic anion transporters 2 and 4 with organic anion transport inhibitors. J Pharmacol Exp Ther 301:797-802.

Hagos Y, Stein D, Ugele B, Burckhardt G, and Bahn A (2007) Human renal organic anion transporter 4 operates as an asymmetric urate transporter. J Am Soc Nephrol 18:430-439.

Hasannejad H, Takeda M, Taki K, Shin HJ, Babu E, Jutabha P, Khamdang S, Aleboyeh M, Onozato ML, Tojo A, et al. (2004) Interactions of human organic anion transporters with diuretics. J Pharmacol Exp Ther 308:1021-1029.

Hnatyshyn S, Shipkova P, and Sanders M (2013) Expedient data mining for nontargeted highresolution LC-MS profiles of biological samples. Bioanalysis 5:1195-1210.

Hsueh CH, Yoshida K, Zhao P, Meyer TW, Zhang L, Huang SM, and Giacomini KM (2016) Identification and quantitative assessment of uremic solutes as inhibitors of renal organic anion transporters, OAT1 and OAT3. Mol Pharm 13:3130-3140.

Imamura Y, Tsuruya Y, Damme K, Heer D, Kumagai Y, Maeda K, Murayama N, Okudaira N, Kurihara A, Izumi T, et al. (2014) $6 \beta$-Hydroxycortisol is an endogenous probe for evaluation of drug-drug interactions involving a multispecific renal organic anion transporter, OAT3/SLC22A8, in healthy subjects. Drug Metab Dispos 42:685-694.

Ito S, Kusuhara H, Kumagai Y, Moriyama Y, Inoue K, Kondo T, Nakayama H, Horita S, Tanabe $\mathrm{K}$, Yuasa $\mathrm{H}$, et al. (2012) $\mathrm{N}$-methylnicotinamide is an endogenous probe for evaluation of drugdrug interactions involving multidrug and toxin extrusions (MATE1 and MATE2-K). Clin Pharmacol Ther 92:635-641.

Jia W, Du F, Liu X, Jiang R, Xu F, Yang J, Li L, Wang F, Olaleye OE, Dong J, et al. (2015) Renal tubular secretion of tanshinol: molecular mechanisms, impact on its systemic exposure, and propensity for dose-related nephrotoxicity and for renal herb-drug interactions. Drug Metab Dispos 43:669-678.

Lacy SA, Hitchcock MJ, Lee WA, Tellier P, and Cundy KC (1998) Effect of oral probenecid coadministration on the chronic toxicity and pharmacokinetics of intravenous cidofovir in cynomolgus monkeys. Toxicol Sci 44:97-106.

Lai Y, Mandlekar S, Shen H, Holenarsipur VK, Langish R, Rajanna P, Murugesan S, Gaud N, Selvam S, Date O, et al. (2016) Coproporphyrins in plasma and urine can be appropriate clinical biomarkers to recapitulate drug-drug interactions mediated by organic anion transporting polypeptide inhibition. J Pharmacol Exp Ther 358:397-404.

Mariappan TT, Shen H, and Marathe P (2017) Endogenous biomarkers to assess drug-drug interactions by drug transporters and enzymes. Curr Drug Metab 18:757-768.

Merrill AH, Jr and Henderson JM (1990) Vitamin B6 metabolism by human liver. Ann N Y Acad Sci 585:110-117.

Mori S, Takanaga H, Ohtsuki S, Deguchi T, Kang YS, Hosoya K, and Terasaki T (2003) Rat organic anion transporter 3 (rOAT3) is responsible for brain-to-blood efflux of homovanillic acid at the abluminal membrane of brain capillary endothelial cells. J Cereb Blood Flow Metab 23:432-440.

Morrissey KM, Stocker SL, Wittwer MB, Xu L, and Giacomini KM (2013) Renal transporters in drug development. Annu Rev Pharmacol Toxicol 53:503-529.

Motohashi H, Sakurai Y, Saito H, Masuda S, Urakami Y, Goto M, Fukatsu A, Ogawa O, and Inui $\mathrm{K}$ (2002) Gene expression levels and immunolocalization of organic ion transporters in the human kidney. J Am Soc Nephrol 13:866-874.

Müller F, Pontones CA, Renner B, Mieth M, Hoier E, Auge D, Maas R, Zolk O, and Fromm MF (2015) N(1)-methylnicotinamide as an endogenous probe for drug interactions by renal cation transporters: studies on the metformin-trimethoprim interaction. Eur J Clin Pharmacol 71:85-94.

Nieskens TT, Peters JG, Schreurs MJ, Smits N, Woestenenk R, Jansen K, van der Made TK, Röring M, Hilgendorf C, Wilmer MJ, et al. (2016) A human renal proximal tubule cell line with stable organic anion transporter 1 and 3 expression predictive for antiviral-induced toxicity. AAPS J 18:465-475.

Nigam SK, Wu W, Bush KT, Hoenig MP, Blantz RC, and Bhatnagar V (2015) Handling of drugs, metabolites, and uremic toxins by kidney proximal tubule drug transporters. Clin J Am Soc Nephrol 10:2039-2049.

Peng CC, Templeton I, Thummel KE, Davis C, Kunze KL, and Isoherranen N (2011) Evaluation of $6 \beta$-hydroxycortisol, $6 \beta$-hydroxycortisone, and a combination of the two as endogenous probes for inhibition of CYP3A4 in vivo. Clin Pharmacol Ther 89:888-895.

Rane A, Villeneuve JP, Stone WJ, Nies AS, Wilkinson GR, and Branch RA (1978) Plasma binding and disposition of furosemide in the nephrotic syndrome and in uremia. Clin Pharmacol Ther $\mathbf{2 4}$ 199-207.

Rodrigues AD, Taskar KS, Kusuhara H, and Sugiyama Y (2017) Endogenous probes for drug transporters: balancing vision with reality. Clin Pharmacol Ther [published ahead of print].

Shen H, Chen W, Drexler DM, Mandlekar S, Holenarsipur VK, Shields EE, Langish R, Sidik K, Gan J, Humphreys WG, et al. (2017) Comparative evaluation of plasma bile acids, dehydroepiandrosterone sulfate, hexadecanedioate, and tetradecanedioate with coproporphyrins I and III as markers of OATP inhibition in healthy subjects. Drug Metab Dispos 45:908-919.

Shen H, Dai J, Liu T, Cheng Y, Chen W, Freeden C, Zhang Y, Humphreys WG, Marathe P, and Lai Y (2016a) Coproporphyrins I and III as functional markers of OATP1B activity: in vitro and in vivo evaluation in preclinical species. J Pharmacol Exp Ther 357:382-393.

Shen H, Liu T, Jiang H, Titsch C, Taylor K, Kandoussi H, Qiu X, Chen C, Sukrutharaj S, Kuit K, et al. (2016b) Cynomolgus monkey as a clinically relevant model to study transport involving renal organic cation transporters: in vitro and in vivo evaluation. Drug Metab Dispos 44:238-249.

Shen H, Yang Z, Zhao W, Zhang Y, and Rodrigues AD (2013) Assessment of vandetanib as an inhibitor of various human renal transporters: inhibition of multidrug and toxin extrusion as a possible mechanism leading to decreased cisplatin and creatinine clearance. Drug Metab Dispos 41:2095-2103.

Smith DE, Gee WL, Brater DC, Lin ET, and Benet LZ (1980) Preliminary evaluation of furosemide-probenecid interaction in humans. J Pharm Sci 69:571-575.

Tahara H, Kusuhara H, Chida M, Fuse E, and Sugiyama Y (2006) Is the monkey an appropriate animal model to examine drug-drug interactions involving renal clearance? Effect of probenecid on the renal elimination of $\mathrm{H} 2$ receptor antagonists. J Pharmacol Exp Ther 316: $1187-1194$

Tahara H, Shono M, Kusuhara H, Kinoshita H, Fuse E, Takadate A, Otagiri M, and Sugiyama Y (2005) Molecular cloning and functional analyses of OAT1 and OAT3 from cynomolgus monkey kidney. Pharm Res 22:647-660.

Thakare R, Gao H, Kosa RE, Bi YA, Varma MVS, Cerny MA, Sharma R, Kuhn M, Huang B, Liu $\mathrm{Y}$, et al. (2017) Leveraging of rifampicin-dosed cynomolgus monkeys to identify bile acid 3-Osulfate conjugates as potential novel biomarkers for organic anion-transporting polypeptides. Drug Metab Dispos 45:721-733.

Tsuruya Y, Kato K, Sano Y, Imamura Y, Maeda K, Kumagai Y, Sugiyama Y, and Kusuhara H (2016) Investigation of endogenous compounds applicable to drug-drug interaction studies involving the renal organic anion transporters, OAT1 and OAT3, in humans. Drug Metab Dispos 44:1925-1933.

Vree TB, van den Biggelaar-Martea M, and Verwey-van Wissen CP (1995) Probenecid inhibits the renal clearance of frusemide and its acyl glucuronide. Br J Clin Pharmacol 39:692-695.

Wikoff WR, Nagle MA, Kouznetsova VL, Tsigelny IF, and Nigam SK (2011) Untargeted metabolomics identifies enterobiome metabolites and putative uremic toxins as substrates of organic anion transporter 1 (Oat1). J Proteome Res 10:2842-2851.

Yee SW, Giacomini MM, Hsueh CH, Weitz D, Liang X, Goswami S, Kinchen JM, Coelho A, Zur AA, Mertsch K, et al. (2016) Metabolomic and genome-wide association studies reveal potential endogenous biomarkers for OATP1B1. Clin Pharmacol Ther 100:524-536.

Zempleni J (1995) Pharmacokinetics of vitamin B6 supplements in humans. J Am Coll Nutr 14 $579-586$.

Address correspondence to: Dr. Hong Shen, Department of Metabolism and Pharmacokinetics, Pharmaceutical Candidate Optimization, Bristol-Myers Squibb Company, Route 206 \& Province Line Road, Princeton, NJ 08543-4000. E-mail: hong.shen1@bms.com 